\title{
Neutrino-electron scattering in dense magnetized plasma
}

\author{
V.G. Bezchastnov \\ A.F. Ioffe Physical-Technical Institute, 194021 St.Petersburg, Russia \\ P. Haensel \\ N. Copernicus Astronomical Center, Polish Academy of Sciences, Bartycka 18, 00-716 Warszawa, Poland
}

(September 20, 2018)

\begin{abstract}
We derive general expressions for the cross section of neutrino scattering on electrons in dense, hot stellar matter, in the presence of strong magnetic fields. Numerical calculations of the scattering cross sections at various densities, temperatures and magnetic fields, are performed. Strong, quantizing magnetic fields modify significantly the angular and energy dependence of the scattering cross section.
\end{abstract}

PACS numbers: 95.30.Cq, 97.60.Bw

to be published in Physical Review D (September, 1996)

\section{INTRODUCTION}

Neutrinos play a crucial role in the gravitational collapse of massive, evolved stellar cores, which is thought to be at the origin of Type II supernova explosions. During the infall phase, when the collapsing stellar core increases its central density from the initial $\sim 10^{10} \mathrm{~g} \mathrm{~cm}^{-3}$ to the supernuclear one, exceeding $10^{14} \mathrm{~g} \mathrm{~cm}^{-3}$, a large number of electron neutrinos is produced, as a result of electron captures on protons (both free protons and those bound in nuclei). The role of neutrinos of other flavors is, during the infall phase, negligible. The fate of electron neutrinos is determined by the opacity of the collapsing core, which, in turn, is determined by their interaction with the dense, hot medium. The main source of neutrino opacity is the elastic scattering on nuclei, which leads to neutrino trapping at $\rho \sim 10^{12}-10^{13} \mathrm{~g} \mathrm{~cm}^{-3}$. Simple estimates suggest that scattering of neutrinos on degenerate electrons is not important as a source of opacity, therefore this process was not included in the earlier collapse simulations (see, e.g., [1] and the references therein). However, as pointed out in [2 4, and confirmed recently in [5], neutrino-electron scattering is a significant source of matter entropy increase as well as of core deleptonization, and therefore its inclusion is important for the reliable determination of the fate of the collapsing core.

The special role of the neutrino-electron scattering (NES),

$$
\nu+e \rightarrow \nu+e,
$$

results from the fact that it can be accompanied by a significant energy transfer, in contrast to the case of neutrinonucleus or neutrino-nucleus scattering, which are to a very good approximation elastic (conservative). Electron capture on free protons (which is a main source of electron neutrinos) produces neutrinos, which are "hotter" than the matter (i.e., their mean energy is significantly larger than that corresponding to matter temperature). As the neutrinos flow from denser to less dense layers of the collapsing core, this excess is even higher than the local one. Energy is transferred from neutrinos to matter mostly by NES, which tends to equilibrate neutrinos and matter. Because on average neutrinos loose energy in the NES, this process decreases the mean neutrino energy, and in consequence, increases its mean free path (the neutrino mean free path scales as the inverse square of the neutrino energy): downscattered neutrinos escape more easily from the collapsing core. On the other hand, neutrino down-scattering heats the matter (increases the matter entropy), which increases the free proton fraction, and this accelerates electron captures. Both effects lead to significantly higher deleptonization of the collapsing core (as compared to the case with NES turned off, see [5]), which turns out to be crucial for the energetics of the post-bounce shock, and to the eventual success or failure of the shock to produce an explosion of the collapsing star.

While observations tell that young neutron stars possess a very strong magnetic field (external magnetic field of radio pulsars $B \sim 10^{12}-10^{13} \mathrm{G}$, the internal magnetic field can be significantly higher, e.g., $B \sim 10^{15} \mathrm{G}$ ), the mechanism of its formation is still a matter of debate. Even less is known about the role of the magnetic field during collapse. The initial (primordial) field of the collapsing core could be greatly amplified due to the huge compression of the matter of very high conductivity. Superstrong magnetic fields $\left(\sim 10^{15} \mathrm{G}\right)$ could also be generated in collapsing and rotating cores ( [6]). If present, such strong magnetic fields could influence NES by modifying the motion 
of electrons in the hot dense plasma. This could lead to anisotropies in the scattering process, implying possible anisotropies and asymmetries in the collapse and explosion.

The problem of NES in a hot, dense plasma in the absence of a magnetic field was studied by numerous authors (see, e.g., [9, 10]). In the present paper we calculate the cross section of this process in the presence of a strong magnetic field. The elementary process we consider here is related to two other weak interaction processes in a hot, dense, magnetized plasma which were considered recently in a paper co-authored by one of the authors ( 11): neutrino-pair synchrotron radiation by electrons, $e^{-} \longrightarrow e^{-}+\nu+\bar{\nu}$, and the neutrino-pair emission from the electron-positron annihilation, $e^{-}+e^{+} \longrightarrow \nu+\bar{\nu}$. The formulae obtained in the present paper complete thus the general study of weak interaction processes involving electrons, positrons, and neutrinos in hot, dense, magnetized plasma.

In Sec. If we briefly present our formalism. The exact calculation of the NES cross section in magnetized plasma is described in Sec. II. In Sec. III we show, using the quasi-classical approximation, convergence of our result to the zero-field limit. Numerical results, obtained for various combinations of hot plasma parameters, neutrino energies, and magnetic field strengths, are presented in Sec. IV. Sec. V contains a discussion of our results and the conclusion. Formulae for the transition amplitude are collected in Appendix A. In Appendix B we rederive a compact expression for the cross section in absence of a magnetic field.In Appendix $\mathrm{C}$ we give an alternative form of the cross section at $B=0$, which proves to be useful in our discussion of the limiting behavior of the cross section at low magnetic field strengths.

\section{GENERAL FORMALISM}

Let us consider NES (11) in a strong uniform magnetic field $\mathbf{B}$ directed along the $z$-axis. We will use the standard Weinberg - Salam - Glashow weak interaction theory. Our results will be valid for all neutrino flavors. Since typical energies of neutrinos, relevant for the infall phase of gravitational collapse, are greater than a few $\mathrm{MeV}$, we can adopt the approximation of massless neutrinos. On the other hand, these typical energies are much smaller than the $m_{\mathrm{W}} c^{2} \sim 70 \mathrm{GeV}$, the rest-mass energy of the intermediate bosons. Therefore the process of scattering (1) can be described by a simplest "four-tail" diagram.

We use exact wave functions of relativistic electrons in a magnetic field, with the Landau gauge of the vector potential A $\left(A_{x}=-y B, A_{y}=A_{z}=0\right)$. We specify the quantum states of electrons by four quantum numbers: $p_{z}, p_{x}, n$, and $s$ (see, e.g., [12]). Here $p_{z}$ is the electron momentum along $\mathbf{B}, p_{x}$ determines the $y$-coordinate of the electron guiding center $y=y_{0}=-c p_{x} /(e B), n=0,1, \ldots$ enumerates the Landau energy levels, and $s$ denotes the electron helicity $\left(s=-\operatorname{sgn}\left(p_{z}\right)\right.$ for $n=0$ and $s= \pm 1$ for $\left.n>0\right)$. We will mostly use the units $k_{\mathrm{B}}=\hbar=c=1$, expressing momenta in units of $m_{e} c$, energies (and temperature) in $m_{e} c^{2}$, and magnetic fields in units of $B_{0}=m_{e}^{2} c^{3} /(\hbar e) \approx 4.414 \times 10^{13} \mathrm{G}$. Then the electron energy is

$$
\varepsilon=\varepsilon_{n}\left(p_{z}\right)=\sqrt{1+2 n b+p_{z}^{2}},
$$

where $b=B / B_{0}$ is the dimensionless magnetic field.

Let us denote the initial state in process (11), corresponding to an electron state $\left|p_{z}, p_{x}, n, s\right\rangle$ and incident neutrino momentum $\mathbf{k}$, by $|\mathbf{i}\rangle$. The final state will be denoted by $|\mathrm{f}\rangle=\left|p_{z}^{\prime}, p_{x}^{\prime}, n^{\prime}, s^{\prime}, \mathbf{k}^{\prime}\right\rangle$. The electron and neutrino energies, corresponding to $|\mathrm{i}\rangle$ and $|\mathrm{f}\rangle$ states, are $\varepsilon, \omega$, and $\varepsilon^{\prime}, \omega^{\prime}$, respectively. The probability of the transition $|\mathrm{i}\rangle \rightarrow|\mathrm{f}\rangle$, per unit time, is given by the Fermi Golden Rule,

$$
\mathrm{d} w_{\mathrm{fi}}=2 \pi \delta\left(\varepsilon^{\prime}+\omega^{\prime}-\varepsilon-\omega\right)\left|V_{\mathrm{fi}}\right|^{2} \mathrm{~d} \rho_{\mathrm{f}},
$$

where

$$
\mathrm{d} \rho_{\mathrm{f}}=\frac{L_{x}}{2 \pi} \mathrm{d} p_{x}^{\prime} \frac{L_{z}}{2 \pi} \mathrm{d} p_{z}^{\prime} \frac{V}{(2 \pi)^{3}} \mathrm{~d} \mathbf{k}^{\prime}
$$

is the density of states of particles after scattering $\left(L_{z}\right.$ and $L_{x}$ are the normalization lengths, and $V$ is the normalization volume), and $V_{\mathrm{f}}$ is the matrix element, calculated for the "four-tail" diagram of process (11).

Calculation of the matrix element is straightforward and analogous to the case of synchrotron neutrino-pair emission $(e \rightarrow e+\nu+\bar{\nu}$; see, e.g., [13]). The final result is

$$
\begin{aligned}
\left|V_{\mathrm{fi}}\right|^{2} & =\frac{(2 \pi)^{2} G_{\mathrm{F}}^{2}}{L_{x} L_{z} V^{2}} \delta\left(p_{x}^{\prime}+k_{x}^{\prime}-p_{x}-k_{x}\right) \\
& \times \delta\left(p_{z}^{\prime}+k_{z}^{\prime}-p_{z}-k_{z}\right) C^{i j} S_{i} S_{j}^{*},
\end{aligned}
$$


where $G_{\mathrm{F}}$ is the Fermi weak-coupling constant, $i$ and $j$ are four-tensor indices $(i, j=0,1,2,3)$,

$$
C^{i j}=\frac{1}{\omega \omega^{\prime}}\left(k^{i} k^{\prime j}+k^{i} k^{j}-g^{i j} k^{l} k_{l}^{\prime}+\mathrm{i} e^{i j l m} k_{l} k_{m}^{\prime}\right)
$$

$k^{i}=(\omega, \mathbf{k}), e^{i j l m}$ is the antisymmetric unit tensor, and $S_{i}$ is the transition amplitude of the four-current for the NES, given by Eq. (A1). We introduce the cross section $\mathrm{d} \sigma_{\mathrm{f} i}=V \mathrm{~d} w_{\mathrm{f} i}$ of the NES by

$$
\mathrm{d} \sigma_{\mathrm{fi}}=\frac{G_{\mathrm{F}}^{2}}{(2 \pi)^{2}} C^{i j} S_{i} S_{j}^{*} \delta\left(\varepsilon^{\prime}+\omega^{\prime}-\varepsilon-\omega\right) \delta\left(p_{x}^{\prime}+k_{x}^{\prime}-p_{x}-k_{x}\right) \delta\left(p_{z}^{\prime}+k_{z}^{\prime}-p_{z}-k_{z}\right) \mathrm{d} p_{x}^{\prime} \mathrm{d} p_{z}^{\prime} \mathrm{d} \mathbf{k}^{\prime}
$$

and perform averaging over initial and summation over final electron states,

$$
\mathrm{d} \sigma=\frac{b}{(2 \pi)^{2}} \sum_{n=0}^{\infty} \int_{-\infty}^{+\infty} \mathrm{d} p_{z} \sum_{n^{\prime}=0}^{\infty} \int_{-\infty}^{+\infty} \mathrm{d} p_{x}^{\prime} \int_{-\infty}^{+\infty} \mathrm{d} p_{z}^{\prime} \sum_{s, s^{\prime}= \pm 1} f\left(1-f^{\prime}\right) \mathrm{d} \sigma_{\mathrm{fi}},
$$

where

$$
f=f(\varepsilon)=\left[\exp \left(\frac{\varepsilon-\mu}{T}\right)+1\right]^{-1}
$$

is the Fermi-Dirac distribution of the electrons in the initial state, while $f^{\prime}=f\left(\varepsilon^{\prime}\right)$ is same quantity for electrons in the final state, $T$ is temperature of matter and $\mu$ is the electron chemical potential (in units of $m_{e} c^{2}$ ). Note that the electron number density (in units of $\left.\left(m_{e} c / \hbar\right)^{3}\right)$ is given by

$$
n_{e}=\frac{b}{(2 \pi)^{2}} \sum_{n=0}^{\infty} \int_{-\infty}^{+\infty} \mathrm{d} p_{z} \sum_{s= \pm 1} f
$$

Taking into account that $\mathrm{d} \mathbf{k}^{\prime}=\omega^{\prime 2} \mathrm{~d} \omega^{\prime} \mathrm{d} \Omega^{\prime}$ ( $\mathrm{d} \Omega^{\prime}$ being a solid angle element along $\mathbf{k}^{\prime}$ ) and introducing the quantities

$$
A_{i j}=\sum_{s, s^{\prime}= \pm 1} S_{i} S_{j}^{*}, \quad D=\varepsilon \varepsilon^{\prime} C^{i j} A_{i j}
$$

we come to the following general expression for the differential cross section of NES in dense magnetized plasma

$$
\begin{aligned}
\frac{\mathrm{d} \sigma}{\mathrm{d} \omega^{\prime} \mathrm{d} \Omega^{\prime}} & =\frac{G_{\mathrm{F}}^{2} b \omega^{\prime 2}}{(2 \pi)^{4}} \sum_{n, n^{\prime}=0}^{\infty} \int_{-\infty}^{+\infty} \frac{\mathrm{d} p_{z}}{\varepsilon \varepsilon^{\prime}} \\
& \times \delta\left(\varepsilon^{\prime}+\omega^{\prime}-\varepsilon-\omega\right) f\left(1-f^{\prime}\right) D .
\end{aligned}
$$

Explicit formulae for the quantities $A_{i j}$ and $D$ are given in Appendix A. These quantities are expressed in terms of the Laguerre functions $F_{n^{\prime}, n}(u)$ defined by Eq. (A5). The Laguerre functions which appear most frequently in the formulae are denoted, for the sake of convenience, as $F_{1}, F_{2}, F_{3}, F_{4}$ : they are defined in (A4).

\section{SCATTERING CROSS SECTION}

Equation (12) expresses the differential scattering cross section as a sum over Landau level numbers of the initial and final electrons and an integral over $p_{z}$. The integrand contains the energy conserving delta function, so that integration over $p_{z}$ can be done analytically. For this purpose, we write

$$
\delta\left(\varepsilon^{\prime}+\omega^{\prime}-\varepsilon-\omega\right)=\sum_{\lambda} \frac{\varepsilon_{\lambda} \varepsilon_{\lambda}^{\prime}}{\left|\varepsilon_{\lambda}^{\prime} p_{z \lambda}-\varepsilon_{\lambda} p_{z \lambda}^{\prime}\right|} \delta\left(p_{z}-p_{z \lambda}\right)
$$

where $\lambda$ enumerates all those ("resonant") sets of energies and longitudinal momenta $\varepsilon, p_{z}, \varepsilon^{\prime}, p_{z}^{\prime}$ which satisfy the conservation laws

$$
\begin{aligned}
\varepsilon^{\prime}+\omega^{\prime} & =\varepsilon+\omega, \\
p_{z}^{\prime}+k_{z}^{\prime} & =p_{z}+k_{z} .
\end{aligned}
$$


It is convenient to introduce the quantities

$$
\begin{aligned}
\Delta & =\omega-\omega^{\prime}, \\
\delta & =k_{z}-k_{z}^{\prime}=\omega \cos \vartheta-\omega^{\prime} \cos \vartheta^{\prime}, \\
\xi & =\Delta^{2}-\delta^{2}=\omega^{2} \sin ^{2} \vartheta+\omega^{\prime 2} \sin ^{2} \vartheta^{\prime} \\
& -2 \omega \omega^{\prime}\left(1-\cos \vartheta \cos \vartheta^{\prime}\right), \\
\chi & =-\xi,
\end{aligned}
$$

where $\vartheta$ and $\vartheta^{\prime}$ are the polar angles of $\mathbf{k}$ and $\mathbf{k}^{\prime}$, respectively. It is also suitable to define the cyclotron harmonic number $\nu=\left|n^{\prime}-n\right|$ in such a way that $\nu \geq 0$. An analysis of possible solutions, $\lambda$, of Eqs. (14), to be used in (13), reveals four different cases, determined by signs of $\Delta$ and $\xi$. We label these cases by A1, A2, B1, and B2, and discuss them below.

Case $\mathbf{A} 1(\Delta \geq 0, \xi \geq 0)$. In this case we always have $n>n^{\prime}$. Eqs. (14) are then satisfied for $\nu \geq \nu_{\min }$ and $n \leq n_{\max }$, where

$$
\nu_{\min }=\operatorname{Int}\left[\frac{\sqrt{\xi}}{b}\left(\frac{\sqrt{\xi}}{2}+1\right)\right]+1, \quad n_{\max }=\operatorname{Int}\left\{\frac{1}{2 b}\left[\left(\frac{\nu b}{\sqrt{\xi}}-\frac{\sqrt{\xi}}{2}\right)^{2}-1\right]\right\},
$$

and where $\operatorname{Int}(a)$ denotes the integral part of $a$. For each $\nu$ and $n$ there are two solutions $\lambda=1,2$ :

$$
\begin{aligned}
\varepsilon_{1} & =\frac{|\Delta|}{\xi}\left(\nu b-\frac{\xi}{2}\right)+\frac{|\delta|}{\xi} \sqrt{\left(\nu b-\frac{\xi}{2}\right)^{2}-\varepsilon_{n 0}^{2} \xi} \\
p_{z 1} & =\left[\frac{|\delta|}{\xi}\left(\nu b-\frac{\xi}{2}\right)+\frac{|\Delta|}{\xi} \sqrt{\left(\nu b-\frac{\xi}{2}\right)^{2}-\varepsilon_{n 0}^{2} \xi}\right] \operatorname{sgn}(\Delta) \operatorname{sgn}(\delta), \\
\varepsilon_{2} & =\frac{\delta^{2} \varepsilon_{n 0}^{2}+\left(\nu b-\frac{\xi}{2}\right)^{2}}{|\Delta|\left(\nu b-\frac{\xi}{2}\right)+|\delta| \sqrt{\left(\nu b-\frac{\xi}{2}\right)^{2}-\varepsilon_{n 0}^{2} \xi}}, \\
p_{z 2} & =\frac{|\delta|\left(\nu b-\frac{\xi}{2}\right)+|\Delta| \sqrt{\left(\nu b-\frac{\xi}{2}\right)^{2}-\varepsilon_{n 0}^{2} \xi}}{\operatorname{sgn}(\Delta) \operatorname{sgn}(\delta),} \\
\varepsilon_{1,2}^{\prime} & =\varepsilon_{1,2}+\Delta, \\
p_{z 1,2}^{\prime} & =p_{z 1,2}+\delta .
\end{aligned}
$$

Final result reads

$$
\frac{\mathrm{d} \sigma}{\mathrm{d} \omega^{\prime} \mathrm{d} \Omega^{\prime}}=\frac{G_{\mathrm{F}}^{2} b}{(2 \pi)^{4}} \omega^{\prime 2} \sum_{\nu=\nu_{\min }}^{\infty} \sum_{n=0}^{n_{\max }} \sum_{\lambda=1,2} \frac{D\left(n, n+\nu, \varepsilon_{\lambda}, \varepsilon_{\lambda}^{\prime}, p_{z \lambda}, p_{z \lambda}^{\prime}\right)}{\sqrt{\left(\nu b-\frac{\xi}{2}\right)^{2}-\varepsilon_{n 0}^{2} \xi}} f\left(\varepsilon_{\lambda}\right)\left[1-f\left(\varepsilon_{\lambda}^{\prime}\right)\right] .
$$

Case A2 $(\Delta \geq 0, \xi<0)$. In this case there are no restrictions on possible values of $n$ and $n^{\prime}$ and there is only a single solution of Eq. (14). However, it is convenient to separate this solution into two sets corresponding to $n \leq n^{\prime}$ and $n>n^{\prime}$ (labeled, respectively, as "1" and "2"):

$$
\begin{aligned}
\varepsilon_{1} & =\frac{\delta^{2} \varepsilon_{n 0}^{2}+\left(\nu b+\frac{\chi}{2}\right)^{2}}{|\Delta|\left(\nu b+\frac{\chi}{2}\right)+|\delta| \sqrt{\left(\nu b+\frac{\chi}{2}\right)^{2}+\varepsilon_{n 0}^{2} \chi}}, \\
p_{z 1}= & \frac{\Delta^{2} \varepsilon_{n 0}^{2}-\left(\nu b+\frac{\chi}{2}\right)^{2}}{|\delta|\left(\nu b+\frac{\chi}{2}\right)+|\Delta| \sqrt{\left(\nu b+\frac{\chi}{2}\right)^{2}+\varepsilon_{n 0}^{2} \chi}} \operatorname{sgn}(\Delta) \operatorname{sgn}(\delta),
\end{aligned}
$$




$$
\begin{aligned}
\varepsilon_{1}^{\prime} & =\varepsilon_{1}+\Delta, \\
p_{z 1}^{\prime} & =p_{z 1}+\delta
\end{aligned}
$$

and

$$
\begin{aligned}
\varepsilon_{2}^{\prime} & =\frac{|\Delta|}{\chi}\left(\nu b+\frac{\chi}{2}\right)+\frac{|\delta|}{\chi} \sqrt{\left(\nu b+\frac{\chi}{2}\right)^{2}+\varepsilon_{n^{\prime} 0}^{2} \chi} \\
p_{z 2}^{\prime} & =\left[\frac{|\delta|}{\chi}\left(\nu b+\frac{\chi}{2}\right)+\frac{|\Delta|}{\chi} \sqrt{\left(\nu b+\frac{\chi}{2}\right)^{2}+\varepsilon_{n^{\prime} 0}^{2} \chi}\right] \operatorname{sgn}(\Delta) \operatorname{sgn}(\delta), \\
\varepsilon_{2} & =\varepsilon_{2}^{\prime}-\Delta \\
p_{z 2} & =p_{z 2}^{\prime}-\delta .
\end{aligned}
$$

The final expression for the cross section can be written as

$$
\begin{aligned}
\frac{\mathrm{d} \sigma}{\mathrm{d} \omega^{\prime} \mathrm{d} \Omega^{\prime}} & =\frac{G_{\mathrm{F}}^{2} b}{(2 \pi)^{4}} \omega^{\prime 2}\left[\sum_{\nu=0}^{\infty} \sum_{n=0}^{\infty} \frac{D\left(n, n+\nu, \varepsilon_{1}, \varepsilon_{1}^{\prime}, p_{z 1}, p_{z 1}^{\prime}\right)}{\sqrt{\left(\nu b+\frac{\chi}{2}\right)^{2}+\varepsilon_{n 0}^{2} \chi}} f\left(\varepsilon_{1}\right)\left[1-f\left(\varepsilon_{1}^{\prime}\right)\right]\right. \\
& \left.+\sum_{\nu=1}^{\infty} \sum_{n^{\prime}=0}^{\infty} \frac{D\left(n^{\prime}+\nu, n^{\prime}, \varepsilon_{2}, \varepsilon_{2}^{\prime}, p_{z 2}, p_{z 2}^{\prime}\right)}{\sqrt{\left(\nu b+\frac{\chi}{2}\right)^{2}+\varepsilon_{n^{\prime} 0}^{2} \chi}} f\left(\varepsilon_{2}\right)\left[1-f\left(\varepsilon_{2}^{\prime}\right)\right]\right]
\end{aligned}
$$

Case B1 $(\Delta<0, \xi \geq 0)$. In this case, only the electron transitions with $n>n^{\prime}$ are allowed. Analogously to the case A1, there exists the lowest cyclotron harmonics, $\nu=\nu_{\min }$, and the highest final Landau level $n^{\prime}=n_{\max }^{\prime}$,

$$
\nu_{\min }=\operatorname{Int}\left[\frac{\sqrt{\xi}}{b}\left(\frac{\sqrt{\xi}}{2}+1\right)\right]+1, \quad n_{\max }^{\prime}=\operatorname{Int}\left\{\frac{1}{2 b}\left[\left(\frac{\nu b}{\sqrt{\xi}}-\frac{\sqrt{\xi}}{2}\right)^{2}-1\right]\right\} .
$$

There are two roots of Eqs. (14) for each $\nu$ and $n^{\prime}$ :

$$
\begin{aligned}
\varepsilon_{1}^{\prime} & =\frac{|\Delta|}{\xi}\left(\nu b-\frac{\xi}{2}\right)+\frac{|\delta|}{\xi} \sqrt{\left(\nu b-\frac{\xi}{2}\right)^{2}-\varepsilon_{n^{\prime} 0}^{2} \xi} \\
p_{z 1}^{\prime} & =\left[\frac{|\delta|}{\xi}\left(\nu b-\frac{\xi}{2}\right)+\frac{|\Delta|}{\xi} \sqrt{\left(\nu b-\frac{\xi}{2}\right)^{2}-\varepsilon_{n^{\prime} 0}^{2} \xi}\right] \operatorname{sgn}(\Delta) \operatorname{sgn}(\delta), \\
\varepsilon_{2}^{\prime} & =\frac{\delta^{2} \varepsilon_{n^{\prime} 0}^{2}+\left(\nu b-\frac{\xi}{2}\right)^{2}}{|\Delta|\left(\nu b-\frac{\xi}{2}\right)+|\delta| \sqrt{\left(\nu b-\frac{\xi}{2}\right)^{2}-\varepsilon_{n^{\prime} 0}^{2} \xi}} \\
p_{z 2}^{\prime} & =\frac{\mid \delta \varepsilon^{2} \varepsilon_{n^{\prime} 0}^{2}-\left(\nu b-\frac{\xi}{2}\right)^{2}}{\xi} \operatorname{sgn}(\Delta) \operatorname{sgn}(\delta), \\
\varepsilon_{1,2} & =\varepsilon_{1,2}^{\prime}-\Delta, \\
p_{z 1,2} & =p_{z 1,2}^{\prime}-\delta
\end{aligned}
$$

The cross section reads

$$
\frac{\mathrm{d} \sigma}{\mathrm{d} \omega^{\prime} \mathrm{d} \Omega^{\prime}}=\frac{G^{2} b}{(2 \pi)^{4}} \omega^{\prime 2} \sum_{\nu=\nu_{\min }}^{\infty} \sum_{n^{\prime}=0}^{n_{\max }^{\prime}} \sum_{\lambda=1,2} \frac{D\left(n^{\prime}+\nu, n^{\prime}, \varepsilon_{\lambda}, \varepsilon_{\lambda}^{\prime}, p_{z \lambda}, p_{z \lambda}^{\prime}\right)}{\sqrt{\left(\nu b-\frac{\xi}{2}\right)^{2}-\varepsilon_{n^{\prime} 0}^{2} \xi}} f\left(\varepsilon_{\lambda}\right)\left[1-f\left(\varepsilon_{\lambda}^{\prime}\right)\right] .
$$


The square-root denominators in Eqs. (18) and (21) for the cases A1 $\left(\nu=n^{\prime}-n\right)$ and B1 $\left(\nu=n-n^{\prime}\right)$, respectively, can be presented in the following symmetric form

$$
\sqrt{\left(\left(n^{\prime}-n\right) b-\frac{\xi}{2}\right)^{2}-\varepsilon_{n 0}^{2} \xi}=\sqrt{\left(\left(n-n^{\prime}\right) b-\frac{\xi}{2}\right)^{2}-\varepsilon_{n^{\prime} 0}^{2} \xi}=\frac{1}{2} \sqrt{\left(\varepsilon_{1}^{2}-\xi\right)\left(\varepsilon_{2}^{2}-\xi\right)},
$$

where $\varepsilon_{1,2}=\left|\varepsilon_{n^{\prime} 0} \mp \varepsilon_{n 0}\right|$. In these cases, the domain of summation over $\nu$ and the lowest Landau level number corresponds to the inequality

$$
\xi \leq \xi_{1}=\varepsilon_{1}^{2}=\frac{4 \nu^{2} b^{2}}{\left(\varepsilon_{n^{\prime} 0}+\varepsilon_{n 0}\right)^{2}},
$$

and the scattering cross section exhibits the square-root singular features when $\xi$ approaches the thresholds $\xi_{1}$, corresponding to the electron transitions $n \rightarrow n^{\prime}=n+\nu$ (case A1) and $n^{\prime} \rightarrow n+\nu$ (case B1). Such features are associated with the behaviour of the density of states for magnetized electron gas and are known to appear for, e.g., electromagnetic cyclotron radiation [14].

Case B2 $(\Delta<0, \xi<0)$. As in the case A2, there are no restrictions on possible values of $n$ and $n^{\prime}$, and single solution of Eqs. (14) can be split into two sets corresponding to $n \leq n^{\prime}$ and $n>n^{\prime}$ (we label them as "1" and "2", respectively):

$$
\begin{aligned}
\varepsilon_{1} & =\frac{|\Delta|}{\chi}\left(\nu b+\frac{\chi}{2}\right)+\frac{|\delta|}{\chi} \sqrt{\left(\nu b+\frac{\chi}{2}\right)^{2}+\varepsilon_{n 0}^{2} \chi} \\
p_{z 1} & =\left[\frac{|\delta|}{\chi}\left(\nu b+\frac{\chi}{2}\right)+\frac{|\Delta|}{\chi} \sqrt{\left(\nu b+\frac{\chi}{2}\right)^{2}+\varepsilon_{n 0}^{2} \chi}\right] \operatorname{sgn}(\Delta) \operatorname{sgn}(\delta), \\
\varepsilon_{1}^{\prime} & =\varepsilon_{1}+\Delta \\
p_{z 1}^{\prime} & =p_{z 1}+\delta
\end{aligned}
$$

and

$$
\begin{aligned}
\varepsilon_{2}^{\prime} & =\frac{\delta^{2} \varepsilon_{n^{\prime} 0}^{2}+\left(\nu b+\frac{\chi}{2}\right)^{2}}{|\Delta|\left(\nu b+\frac{\chi}{2}\right)+|\delta| \sqrt{\left(\nu b+\frac{\chi}{2}\right)^{2}+\varepsilon_{n^{\prime} 0}^{2} \chi}}, \\
p_{z 2}^{\prime} & =\frac{\Delta^{2} \varepsilon_{n^{\prime} 0}^{2}-\left(\nu b+\frac{\chi}{2}\right)^{2}}{|\delta|\left(\nu b+\frac{\chi}{2}\right)+|\Delta| \sqrt{\left(\nu b+\frac{\chi}{2}\right)^{2}+\varepsilon_{n^{\prime} 0}^{2} \chi}} \operatorname{sgn}(\Delta) \operatorname{sgn}(\delta), \\
\varepsilon_{2}= & \varepsilon_{1}^{\prime}-\Delta, \\
p_{z 2}= & p_{z 2}^{\prime}-\delta .
\end{aligned}
$$

The cross section can then be written as

$$
\begin{aligned}
\frac{\mathrm{d} \sigma}{\mathrm{d} \omega^{\prime} \mathrm{d} \Omega^{\prime}} & =\frac{G^{2} b}{(2 \pi)^{4}} \omega^{\prime 2}\left[\sum_{\nu=1}^{\infty} \sum_{n=0}^{\infty} \frac{D\left(n, n+\nu, \varepsilon_{1}, \varepsilon_{1}^{\prime}, p_{z 1}, p_{z 1}^{\prime}\right)}{\sqrt{\left(\nu b+\frac{\chi}{2}\right)^{2}+\varepsilon_{n 0}^{2} \chi}} f\left(\varepsilon_{1}\right)\left[1-f\left(\varepsilon_{1}^{\prime}\right)\right]\right. \\
& \left.+\sum_{\nu=0}^{\infty} \sum_{n^{\prime}=0}^{\infty} \frac{D\left(n^{\prime}+\nu, n^{\prime}, \varepsilon_{2}, \varepsilon_{2}^{\prime}, p_{z 2}, p_{z 2}^{\prime}\right)}{\sqrt{\left(\nu b+\frac{\chi}{2}\right)^{2}+\varepsilon_{n^{\prime} 0}^{2} \chi}} f\left(\varepsilon_{2}\right)\left[1-f\left(\varepsilon_{2}^{\prime}\right)\right]\right] .
\end{aligned}
$$

Similar to Eq. (25), the square-root denominators in Eqs. (21) and (29) can also be written in the symmetric form

$$
\sqrt{\left(\left(n^{\prime}-n\right) b+\frac{\chi}{2}\right)^{2}-\varepsilon_{n 0}^{2} \chi}=\sqrt{\left(\left(n-n^{\prime}\right) b+\frac{\chi}{2}\right)^{2}-\varepsilon_{n^{\prime} 0}^{2} \chi}=\frac{1}{2} \sqrt{\left(\varepsilon_{1}^{2}+\chi\right)\left(\varepsilon_{2}^{2}+\chi\right)} .
$$


Note that, in the case A2 (B2), the contributions from the transitions with $n^{\prime} \leq n\left(n \leq n^{\prime}\right)$ decrease with decreasing $\chi$, and vanish at $\chi=0$. In each case, for $\xi=\chi=0$, some resonant electron states have infinite energies and therefore do not contribute to the cross section, but there are always the resonant states with finite energies. Therefore, for $\xi=\chi=0$, expressions obtained in the cases A1 and A2 (B1 and B2) give the same results for the scattering cross section.

If $\Delta=0$, then $\xi$ can take only non-positive values, and Eqs. (21) and (29) derived for the cases A2 and B2, respectively, coincide.

\section{ZERO FIELD LIMIT}

The neutrino-electron scattering for $B=0$ has been considered by numerous authors (see, e.g., [5, 9, 15], and the references therein). For a degenerate electron gas, the scattering cross section can be then presented in a quite compact form involving standard Fermi integrals. In view of the fact that we could not find in the literature a complete and convincing derivation of the $B=0$ expression for the scattering cross section, we preferred to rederive it in Appendix B. Of course, this expression for the scattering cross section should be re-obtained as a limit of the expressions presented in Section II in the case when the quantization of the electron motion in the plane perpendicular to $\mathbf{B}$ becomes negligible. The effect of the magnetic field is small, when the energy difference between neighboring Landau levels is much smaller than the temperature of the matter, $\varepsilon_{n+1,0}-\varepsilon_{n, 0} \ll T$. Since $\varepsilon_{n+1,0}-\varepsilon_{n, 0}=2 b /\left(\varepsilon_{n+1,0}+\varepsilon_{n, 0}\right)$, and the energies of the most populated electron states can be approximated by the electron chemical potential (assuming $\mu \gg 1), \varepsilon_{n+1.0} \sim \varepsilon_{n, 0} \sim \mu$, we arrive at the condition $b \ll \mu T$. Under such a condition, the expressions given by Eqs. (18), (21), (24), and (29) should converge to the $B=0$ expressions given by Eqs. (B1) and (B23).

Analytical demonstration of such a convergence is quite complicated, due to the strongly different forms of final expressions for the scattering cross section in the case of a magnetized plasma (Section III) and in the $B=0$ case (Appendix B), respectively. We will prove that our general equation (12) reproduces correctly the limit of $B \rightarrow 0$. To facilitate the proof, we transform the expressions for $B=0$, introducing an arbitrary $z$-axis and rearranging the order of integration over initial and final momenta of the electron in the corresponding cylindrical coordinates (see Appendix C).

The zero field limit of Eq. (12) can be obtained using the quasi-classical expressions for the Laguerre functions $F_{n^{\prime} n}(u)$ which enter the quantity $D$ (Appendix A). In our limiting case, high Landau levels are involved, $n \gg 1$ and $n^{\prime} \gg 1$, and we can neglect the difference between $F_{1}^{2}, F_{2}^{2}, F_{3}^{2}$ and $F_{4}^{2}$. All these functions are then equal to (see, e.g., [12])

$$
F^{2}=\frac{2 b}{\pi \sqrt{\left(p_{1}^{2}-q_{\perp}^{2}\right)\left(q_{\perp}^{2}-p_{2}^{2}\right)}}
$$

where $p_{1,2}=p_{\perp}^{\prime} \pm p_{\perp}$. Then, using the recurrent relations for the Laguerre functions [12], we have

$$
\begin{aligned}
p_{\perp} p_{\perp}^{\prime} F_{1} F_{2} & =\frac{\left(p_{\perp}^{2}-p_{\perp}^{\prime 2}\right)^{2}-\left(p_{\perp}^{2}+p_{\perp}^{\prime 2}\right) q_{\perp}^{2}}{2 q_{\perp}^{2}} F^{2}, \\
p_{\perp} p_{\perp}^{\prime} F_{3} F_{4} & =\frac{p_{\perp}^{2}+p_{\perp}^{\prime 2}-q_{\perp}^{2}}{2} F^{2}, \\
p_{\perp}\left(F_{1} F_{3}+F_{2} F_{4}\right) & =\frac{p_{\perp}^{2}-p_{\perp}^{\prime 2}+q_{\perp}^{2}}{q_{\perp}} F^{2}, \\
p_{\perp}^{\prime}\left(F_{1} F_{4}+F_{2} F_{3}\right) & =\frac{p_{\perp}^{2}-p_{\perp}^{\prime 2}-q_{\perp}^{2}}{q_{\perp}} F^{2}, \\
p_{\perp}\left(F_{1} F_{3}-F_{2} F_{4}\right) & =p_{\perp}^{\prime}\left(F_{1} F_{4}-F_{2} F_{3}\right)=0 .
\end{aligned}
$$

Substitution of Eqs. (31) and (32) into Eq. (A7) gives the quasi-classical expression (C19) for the quantity $Q=$ $D /\left(2 F^{2}\right)$. Finally, replacing the summation over $n$ and $n^{\prime}$ in $(12)$ by integration over $p_{\perp}^{2}$ and $p_{\perp}^{\prime 2}$,

$$
\sum_{n, n^{\prime}} \rightarrow \iint \frac{\mathrm{d} p_{\perp}^{2} \mathrm{~d} p_{\perp}^{\prime 2}}{4 b^{2}},
$$

we get Eq. ( $\overline{\mathrm{C} 18})$, obtained for the case of zero magnetic field. This strictly proves that our expression for the cross section of neutrino scattering on magnetized electrons, Eq. (12), tends to the familiar cross section in the limit of $B \rightarrow 0$. 


\section{NUMERICAL RESULTS}

We have calculated the NES cross section using the expressions presented in Section II, for a broad choice of physical conditions in dense, hot, magnetized plasma. These conditions were determined by the values of the dimensionless parameters $\mu, T$, and $b$. The calculations were done for the scattering of electron neutrino $\left(C_{V} \approx 0.96, C_{A}=0.5\right)$, although the general expressions of Section II can be applied for the scattering of neutrino of any flavor.

We have used a simple approximate treatment of the singularities, appearing in cases A1 and B1. Namely, when calculating the square-root denominators according to Eq. (25), we were making the replacement

$$
\varepsilon_{1}^{2}-\xi \rightarrow\left(\varepsilon_{1}+\sqrt{\xi}\right) \sqrt{\left(\varepsilon_{1}-\sqrt{\xi}\right)^{2}+\gamma^{2}}
$$

Here, $\gamma$ has the meaning of a width of the electron energy levels, determined by an appropriate mechanism of deexcitation of the electron states. For the interior of a neutron star, the most important are the electron-nucleus collisions. Assuming that the electrons are degenerate and neglecting the quantization of their transverse motion, we may estimate the widths by the well-known result for the frequency of the electron-nucleus Coulomb collisions 16

$$
\gamma_{\text {coll }} \approx \frac{4 \alpha_{\mathrm{f}}^{2}}{3 \pi} Z \mu
$$

where $\alpha_{\mathrm{f}}=1 / 137$ is the fine structure constant, $Z$ is the nucleus charge number, and $\mu$ is the electron chemical potential. In the range of temperatures and densities, for which NES is most important during the infall stage of gravitational collapse $\left(k_{\mathrm{B}} T \sim 1-2 \mathrm{MeV}, \rho \sim 10^{10}-10^{12} \mathrm{~g} \mathrm{~cm}^{-3}\right.$, see 河), we expect $Z \sim 30-35$.

In order to visualize the different domains of the NES in a magnetized plasma, which were discussed in Section [II (cases A1, A2, B1, and B2), we present in Fig. 1 the differential cross section for this process as a function of the initial neutrino energy. The bottom panel of this figure shows the dependence of the parameter $\xi$ on the energy transfer. We also show the threshold values $\xi_{1}$ associated with the electron transition from $\left(\omega>\omega^{\prime}\right.$, case A1) or to $\left(\omega<\omega^{\prime}\right.$, case B1) the ground Landau level for different cyclotron harmonic numbers $\nu$, and connect them with the corresponding seesaw-like peaks, associated with the square-root singularities in the electron density of states.

It should also be noted, that peaks of another kind appear in the cross section near the boundaries separating the domains A1 and A2, and B1 and B2, respectively. They are associated with those "resonant" electron energies which grow infinitely at $\xi$ approaching zero. For the cases A1 and B1, these energies are labeled by "2" and "1", respectively (see Eqs. (17) and (23)). For the domain of $\xi<0$, the energies of the electron transitions with $n^{\prime}=n(\nu=0)$ become infinite at $\xi \rightarrow 0$; they are labeled by "1" and "2" for the cases A2 and B2, respectively (see Eqs (21) and (29)). Since with increasing energy the cross section grows as energy squared, the above discussed features produce narrow peaks with amplitudes determined by the condition $\varepsilon \approx \mu$ or $\varepsilon^{\prime} \approx \mu$. The peak shapes are also determined by the Fermi-Dirac distribution of electrons: note that the factor $f\left(1-f^{\prime}\right)$ is a peaked function of initial (final) neutrino energy at the low energy transfer $(|\Delta| \lesssim T)$. Two examples of such peaks are seen in Fig. 2, they are associated there with the electron transitions $n=2 \rightarrow n^{\prime}=1$ (case B1), and $n=1 \rightarrow n^{\prime}=1$ (case B2).

A sequence of plots in Fig. 3 shows the convergence of the scattering cross section to the zero field limit. Calculations were performed for different strengths of the magnetic field, at fixed values of the electron chemical potential and temperature which are similar to those prevailing in the outer shells of a collapsing core where NES is important. The initial neutrino energies were also chosen to be similar to the mean neutrino energies in these shells (see [5]). With the decrease of the magnetic field, the quantum oscillations of the cross section become less pronounced, and deviations of its low and high initial neutrino energy tails from the zero field limit (see left panel of Fig. (3) disappear.

At $B=0$, the geometry of the NES can be described by only one parameter, which is the scattering angle $\theta$ between the neutrino momenta, $\mathbf{k}^{\prime}$ and $\mathbf{k}$. At presence of the magnetic field, the geometry is more complicated, since $\theta$ is determined by three independent variables, $\cos \theta=\cos \vartheta \cos \vartheta^{\prime}+\sin \vartheta \sin \vartheta^{\prime} \cos \left(\alpha-\alpha^{\prime}\right)$. The cross section becomes then quite sensitive to a choice of $\vartheta$ and $\vartheta^{\prime}$. In particular, these angles determine the boundaries of the domain A2+B2. Denoting them by $\omega_{\mathrm{r}, 1}$ (at fixed $\omega^{\prime}$ ) and by $\omega_{\mathrm{r}, 1}^{\prime}$ (at fixed $\omega$ ), we can find from the condition $\xi=0$ the following relation,

$$
\begin{aligned}
\sin ^{2} \vartheta \frac{\omega_{\mathrm{r}, 1}}{\omega^{\prime}} & =\sin ^{2} \vartheta^{\prime} \frac{\omega_{\mathrm{r}, 1}^{\prime}}{\omega}=1-\cos \vartheta \cos \vartheta^{\prime} \\
& \pm \sqrt{\left(1-\cos \left(\vartheta-\vartheta^{\prime}\right)\right)\left(1+\cos \left(\vartheta+\vartheta^{\prime}\right)\right)}
\end{aligned}
$$

Note that the lower and the upper boundaries coincide at $\vartheta=\vartheta^{\prime}$ or $\vartheta=-\vartheta^{\prime}$.

In Figs. 1 and 5 we present the cross section for the scattering angle $\theta=90^{\circ}$, for two limiting cases, when the scattered neutrino propagates in the plane "k-B", or in the direction perpendicular to this plane, respectively. We 
have calculated, under these kinematical conditions, the cross section as the function of $\omega^{\prime}$ at different polar angles $\vartheta$ of the incident neutrino.

In Fig. 1, eight examples of the cross section versus energy plots are shown, which correspond to $\theta=90^{\circ}$, and for $\vartheta$ varying from zero to $315^{\circ}$ (with steps of $45^{\circ}$ ). As we see, the shapes of all cross section plots are strikingly different. The dependence of the cross section on $\vartheta$ in the case of the scattering in the plane " $\mathbf{k}-\mathbf{B}$ " has the periodicity of $360^{\circ}$, since at fixed scattering angle different $\vartheta$ correspond to different $\vartheta^{\prime}$, while the cross section is determined by both polar angles. Let us notice specific features of the plots for $\vartheta=0$ and $\vartheta=180^{\circ}$, where the domain $\mathrm{A} 2+\mathrm{B} 2$ in the cross section is the widest $\left(\omega_{1}^{\prime}=0, \omega_{\mathrm{r}}^{\prime}=2 \omega\right)$, and the plots for $\vartheta=135^{\circ}$ and $\vartheta=315^{\circ}$, when this domain vanishes completely. For $\vartheta=135^{\circ}$ and $\vartheta=315^{\circ}$, both $\Delta$ and $\xi$ are equal to zero at the boundary of the domains A1 and B1, so that all the "resonant" electron energies become infinite (see Eqs. (17) and (23)). This leads to the deep minima in the cross section around the point $\omega^{\prime}=\omega$, at which the cross section is strictly equal to zero.

When the scattered neutrino propagates transversely to $\mathbf{B}$ and the angle of scattering, $\theta$, is fixed, the shape of the cross section is determined only by the polar angle of the incident neutrino. In this case, the cross section is invariant with respect to the replacement of $\vartheta$ by $360^{\circ}-\vartheta$, which is demonstrated in Fig. 5 . One can notice that the influence of magnetic field on the cross section is most significant at $\vartheta=90^{\circ}$, where the quantum (oscillatory) features appear near the maximum of the cross section, and the domain without quantum singularities $(\mathrm{A} 2+\mathrm{B} 2)$ vanishes. Comparison of this example with those shown in Fig. 1 allows one to conclude, that the quantum oscillations are most pronounced in the cross section, when both incident and scattered neutrino move transversely to the strong magnetic field.

To conclude our analysis, we show in Fig. 6 how the presence of the magnetic field breaks the invariance of cross the section with respect to the exchange of directions of $\mathbf{k}$ and $\mathbf{k}^{\prime}$. We have set $\alpha=\alpha^{\prime}=45^{\circ}$ and computed the cross section for different pairs of $\vartheta \leftrightarrow \vartheta^{\prime}$. One can see that the regions of prominent quantum oscillations are different for all pairs $\vartheta \leftrightarrow \vartheta^{\prime}$. On the other hand, one can notice that the effect of anisotropy is correlated with the specific direction of the propagation of one of the neutrinos (incident or scattered) relative to the magnetic field. Namely, in the case when the initial (final) neutrino momentum is parallel to $\mathbf{B}$, there is a strong decrease of the cross section for the neutrino energy loss (Fig. [a). This effect is reversed (increase of cross section for energy loss), if the initial or final neutrino momentum is anti-parallel to $\mathbf{B}$ (Fig. [b bee also examples shown in Fig. (4)).

Numerical results, presented in this section, were obtained for selected sets of the input parameters entering the expressions for the scattering cross section. These results were used to show some characteristic features of the neutrino-electron scattering in a hot, dense magnetized plasma. Both the formulae and numerical results are quite complex. Therefore, to make our results usable for interested workers in this field, the Fortran program is available upon request from one of the authors (V.G.Bezchastnov, e-mail: vit@astro.ioffe.rssi.ru).

\section{DISCUSSION AND CONCLUSIONS}

We have considered the NES in a dense, hot, degenerate plasma, under conditions expected to prevail during the infall phase of the gravitational collapse, and assuming the presence of a strong magnetic field. Our results show that the magnetic field modifies the energy dependence of the scattering cross section, and introduces an anisotropy correlated with the direction of $\mathbf{B}$. The quantization of the electron motion induces the appearance of an oscillatory behavior of the cross section as a function of the energy transfer. The amplitude of the oscillations and the distance between them strongly depend on the magnetic field strength.

Among the neutrino processes which are relevant for the infall phase of gravitational collapse, the neutrino-electron scattering is the most sensitive one to the presence of a strong magnetic field. It should be noted, however, that a very strong magnetic field, exceeding $10^{15} \mathrm{G}$, influences also the rate of electron captures on protons, which produce electron neutrinos during the infall stage. Generally, the presence of a strong magnetic field decreases the rate of electron captures [17]. However, this effect can be neglected for the densities greater than $10^{10} \mathrm{~g} \mathrm{~cm}^{-3}$, and therefore will not play a significant role for the collapse scenario, where the electron captures take place mainly at the density close to the trapping density.

The presence of a strong magnetic field implies a very strong sensitivity of the differential cross section for the NES on the spatial direction of neutrino momenta, as well as on the energy transfer. This could lead to anisotropies and asymmetry in the heating of matter, due to anisotropic energy transfer from neutrinos to matter, as well as to the anisotropic and/or asymmetric momentum transfer to the matter. Both effects could contribute to asymmetry and anisotropy of the subsequent explosion of the outer layers of collapsing core.

Both anisotropy and sensitivity to the energy transfer become dramatically large for a sufficiently strong magnetic field, which by itself could influence the dynamics of collapsing layers of the stellar core. Let us define the limiting

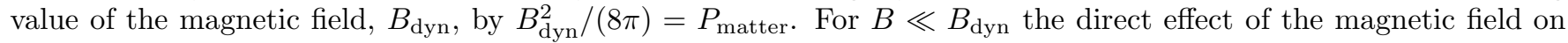
the dynamics of a layer can be neglected, while for $B \gtrsim B_{\mathrm{dyn}}$ the magnetic field modifies in an essential way the 
equation of state of a given layer of the collapsing core. We have $B_{\text {dyn }}=5 \times 10^{15}\left(P_{\text {matter }} / 10^{30} \mathrm{dyn}_{\mathrm{cm}}^{-2}\right)^{1 / 2} \mathrm{G}$. Using the results of numerical simulations of [5], we find that for the layers in which the neutrino-electron scattering is important, $B_{\mathrm{dyn}} \sim 10^{15} \mathrm{G}$. Our numerical results show, that the effects of the magnetic field on the NES become very strong for a field strength of the order of $B_{\mathrm{dyn}}$. It should be mentioned, however, that the fact that the local magnetic field strength exceeds $B_{\text {dyn }}$ does not mean that the magnetic field influences in a crucial way the dynamics of collapse: the effect of the local pressure on the dynamics of collapsing outer layers is not very important during the infall phase.

\section{ACKNOWLEDGMENTS}

This work was supported in part by the Polish State Committee for Scientific Research (KBN), grant No. P304 014 07, as well as by the INTAS grant No. 94-3834 and Soros grant No. R6A000. One of the authors (V.G.B.) is grateful for excellent conditions and hospitality during his stay at the N. Copernicus Astronomical Center. We express our gratitude to D.G. Yakovlev for useful discussions and for helpful remarks concerning the analytical part of this work.

\section{APPENDIX A:}

The structure of the transition amplitude of the four-current $S^{i}=\left(S^{0}, \mathbf{S}\right)$ for the NES is similar to that for the neutrino synchrotron process (see, e.g., [11,13]). We have

$$
\begin{aligned}
S^{0} & =\left[C_{V}\left(\alpha \alpha^{\prime}+s s^{\prime} \beta \beta^{\prime}\right)-C_{A}\left(\alpha s^{\prime} \beta^{\prime}+\alpha^{\prime} s \beta\right)\right] a^{0}, \\
\mathbf{S} & =\left[C_{V}\left(\alpha s^{\prime} \beta^{\prime}+\alpha^{\prime} s \beta\right)-C_{A}\left(\alpha \alpha^{\prime}+s s^{\prime} \beta \beta^{\prime}\right)\right] \mathbf{a},
\end{aligned}
$$

where

$$
\begin{aligned}
& a^{0}=A A^{\prime} F_{3}+s s^{\prime} B B^{\prime} F_{4}, \\
& a^{1}=A^{\prime} B s e^{\mathrm{i} \varphi} F_{1}+s^{\prime} B^{\prime} A e^{-\mathrm{i} \varphi} F_{2}, \\
& a^{2}=-i A^{\prime} B s e^{\mathrm{i} \varphi} F_{1}+i s^{\prime} B^{\prime} A e^{-\mathrm{i} \varphi} F_{2}, \\
& a^{3}=A A^{\prime} F_{3}-s s^{\prime} B B^{\prime} F_{4} .
\end{aligned}
$$

In the above formulae,

$$
\begin{gathered}
\left(\begin{array}{c}
\alpha \\
\beta
\end{array}\right)=\frac{1}{\sqrt{2}}\left(1 \pm \frac{1}{\varepsilon}\right)^{1 / 2}, \\
\left(\begin{array}{c}
A \\
B
\end{array}\right)=\frac{1}{\sqrt{2}}\left(1 \pm \frac{s p_{z}}{\sqrt{\varepsilon^{2}-1}}\right)^{1 / 2}, \\
F_{1}=F_{n^{\prime}-1, n}(u), \quad F_{2}=F_{n^{\prime}, n-1}(u), \\
F_{3}=F_{n^{\prime}-1, n-1}(u), \quad F_{4}=F_{n^{\prime}, n}(u),
\end{gathered}
$$

and

$$
F_{n^{\prime}, n}(u)=\left(\frac{n^{\prime} !}{n !} u^{n-n^{\prime}}\right)^{1 / 2} \mathrm{e}^{-u / 2} L_{n^{\prime}}^{n-n^{\prime}}(u)
$$

is a Laguerre function, $L_{p}^{q}(u)$ being an associated Laguerre polynomial. In Eq. (A1) $C_{V}$ and $C_{A}$ are the vector and axial vector weak interaction constants, respectively. For the scattering of electron neutrino (via charged and neutral currents), one has $C_{V}=2 \sin ^{2} \theta_{W}+0.5$ and $C_{A}=0.5$, while for the scattering of muonic or tauonic neutrinos (neutral currents only), $C_{V}^{\prime}=2 \sin ^{2} \theta_{W}-0.5$ and $C_{A}^{\prime}=-0.5$. Here $\theta_{W}$ is the Weinberg angle, $\sin ^{2} \theta_{W} \simeq 0.23$.

The only difference with respect to the case of the neutrino synchrotron radiation, is that in our case $\varphi$ in Eq. (A2) is an angle between $\mathbf{k}_{\perp}^{\prime}-\mathbf{k}_{\perp}$ and the $x$-axis, and the argument of the Laguerre functions is $u=\left(\mathbf{k}_{\perp}^{\prime}-\mathbf{k}_{\perp}\right)^{2} /(2 b)$.

Calculation of the 4 -tensor $A_{i j}$ yields: 


$$
\begin{aligned}
& A_{00}+A_{33}=\left(C_{V}^{2}+C_{A}^{2}\right)\left(1+\frac{p_{z} p_{z}^{\prime}}{\varepsilon \varepsilon^{\prime}}\right)\left(F_{3}^{2}+F_{4}^{2}\right)-2 C_{V} C_{A}\left(\frac{p_{z}}{\varepsilon}+\frac{p_{z}^{\prime}}{\varepsilon^{\prime}}\right)\left(F_{3}^{2}-F_{4}^{2}\right), \\
& A_{00}-A_{33}=2\left(C_{V}^{2}+C_{A}^{2}\right) \frac{p_{\perp} p_{\perp}^{\prime}}{\varepsilon \varepsilon^{\prime}} F_{3} F_{4}+\frac{C_{V}^{2}-C_{A}^{2}}{\varepsilon \varepsilon^{\prime}}\left(F_{3}^{2}+F_{4}^{2}\right), \\
& A_{11}+A_{22}=\left(C_{V}^{2}+C_{A}^{2}\right)\left(1-\frac{p_{z} p_{z}^{\prime}}{\varepsilon \varepsilon^{\prime}}\right)\left(F_{1}^{2}+F_{2}^{2}\right)-\frac{C_{V}^{2}-C_{A}^{2}}{\varepsilon \varepsilon^{\prime}}\left(F_{1}^{2}+F_{2}^{2}\right)+2 C_{V} C_{A}\left(\frac{p_{z}}{\varepsilon}-\frac{p_{z}^{\prime}}{\varepsilon^{\prime}}\right)\left(F_{1}^{2}-F_{2}^{2}\right), \\
& A_{11}-A_{22}=2\left(C_{V}^{2}+C_{A}^{2}\right) \frac{p_{\perp} p_{\perp}^{\prime}}{\varepsilon \varepsilon^{\prime}} \cos (2 \varphi) F_{1} F_{2} \\
& \frac{A_{01}+A_{10}}{\cos \varphi}=-\left(C_{V}^{2}+C_{A}^{2}\right)\left[\frac{p_{\perp}}{\varepsilon}\left(F_{1} F_{3}+F_{2} F_{4}\right)+\frac{p_{\perp}^{\prime}}{\varepsilon^{\prime}}\left(F_{1} F_{4}+F_{2} F_{3}\right)\right] \\
& +2 C_{V} C_{A}\left[\frac{p_{\perp} p_{z}^{\prime}}{\varepsilon \varepsilon^{\prime}}\left(F_{1} F_{3}-F_{2} F_{4}\right)-\frac{p_{\perp}^{\prime} p_{z}}{\varepsilon \varepsilon^{\prime}}\left(F_{1} F_{4}-F_{2} F_{3}\right)\right], \\
& \frac{A_{01}-A_{10}}{\mathrm{i} \sin \varphi}=\left(C_{V}^{2}+C_{A}^{2}\right)\left[\frac{p_{\perp}}{\varepsilon}\left(F_{1} F_{3}-F_{2} F_{4}\right)+\frac{p_{\perp}^{\prime}}{\varepsilon^{\prime}}\left(F_{1} F_{4}-F_{2} F_{3}\right)\right] \\
& -2 C_{V} C_{A}\left[\frac{p_{\perp} p_{z}^{\prime}}{\varepsilon \varepsilon^{\prime}}\left(F_{1} F_{3}+F_{2} F_{4}\right)-\frac{p_{\perp}^{\prime} p_{z}}{\varepsilon \varepsilon^{\prime}}\left(F_{1} F_{4}+F_{2} F_{3}\right)\right], \\
& \frac{A_{02}+A_{20}}{\sin \varphi}=-\left(C_{V}^{2}+C_{A}^{2}\right)\left[\frac{p_{\perp}}{\varepsilon}\left(F_{1} F_{3}+F_{2} F_{4}\right)+\frac{p_{\perp}^{\prime}}{\varepsilon^{\prime}}\left(F_{1} F_{4}+F_{2} F_{3}\right)\right] \\
& +2 C_{V} C_{A}\left[\frac{p_{\perp} p_{z}^{\prime}}{\varepsilon \varepsilon^{\prime}}\left(F_{1} F_{3}-F_{2} F_{4}\right)-\frac{p_{\perp}^{\prime} p_{z}}{\varepsilon \varepsilon^{\prime}}\left(F_{1} F_{4}-F_{2} F_{3}\right)\right], \\
& \frac{A_{02}-A_{20}}{\mathrm{i} \cos \varphi}=-\left(C_{V}^{2}+C_{A}^{2}\right)\left[\frac{p_{\perp}}{\varepsilon}\left(F_{1} F_{3}-F_{2} F_{4}\right)+\frac{p_{\perp}^{\prime}}{\varepsilon^{\prime}}\left(F_{1} F_{4}-F_{2} F_{3}\right)\right] \\
& +2 C_{V} C_{A}\left[\frac{p_{\perp} p_{z}^{\prime}}{\varepsilon \varepsilon^{\prime}}\left(F_{1} F_{3}+F_{2} F_{4}\right)-\frac{p_{\perp}^{\prime} p_{z}}{\varepsilon \varepsilon^{\prime}}\left(F_{1} F_{4}+F_{2} F_{3}\right)\right], \\
& A_{03}+A_{30}=-\left(C_{V}^{2}+C_{A}^{2}\right)\left(\frac{p_{z}}{\varepsilon}+\frac{p_{z}^{\prime}}{\varepsilon^{\prime}}\right)\left(F_{3}^{2}+F_{4}^{2}\right)+2 C_{V} C_{A}\left(1+\frac{p_{z} p_{z}^{\prime}}{\varepsilon \varepsilon^{\prime}}\right)\left(F_{3}^{2}-F_{4}^{2}\right), \\
& A_{03}-A_{30}=0 \text {, } \\
& A_{12}+A_{21}=2 \sin (2 \varphi)\left(C_{V}^{2}+C_{A}^{2}\right) \frac{p_{\perp} p_{\perp}^{\prime}}{\varepsilon \varepsilon^{\prime}} F_{1} F_{2}, \\
& \frac{A_{12}-A_{21}}{\mathrm{i}}=\left(C_{V}^{2}+C_{A}^{2}\right)\left(1-\frac{p_{z} p_{z}^{\prime}}{\varepsilon \varepsilon^{\prime}}\right)\left(F_{1}^{2}-F_{2}^{2}\right)-\frac{C_{V}^{2}-C_{A}^{2}}{\varepsilon \varepsilon^{\prime}}\left(F_{1}^{2}-F_{2}^{2}\right)+2 C_{V} C_{A}\left(\frac{p_{z}}{\varepsilon}-\frac{p_{z}^{\prime}}{\varepsilon^{\prime}}\right)\left(F_{1}^{2}+F_{2}^{2}\right) \text {, } \\
& \frac{A_{13}+A_{31}}{\cos \varphi}=\left(C_{V}^{2}+C_{A}^{2}\right)\left[\frac{p_{\perp} p_{z}^{\prime}}{\varepsilon \varepsilon^{\prime}}\left(F_{1} F_{3}+F_{2} F_{4}\right)+\frac{p_{\perp}^{\prime} p_{z}}{\varepsilon \varepsilon^{\prime}}\left(F_{1} F_{4}+F_{2} F_{3}\right)\right] \\
& -2 C_{V} C_{A}\left[\frac{p_{\perp}}{\varepsilon}\left(F_{1} F_{3}-F_{2} F_{4}\right)-\frac{p_{\perp}^{\prime}}{\varepsilon^{\prime}}\left(F_{1} F_{4}-F_{2} F_{3}\right)\right] \text {, } \\
& \frac{A_{13}-A_{31}}{\mathrm{i} \sin \varphi}=\left(C_{V}^{2}+C_{A}^{2}\right)\left[\frac{p_{\perp} p_{z}^{\prime}}{\varepsilon \varepsilon^{\prime}}\left(F_{1} F_{3}-F_{2} F_{4}\right)+\frac{p_{\perp}^{\prime} p_{z}}{\varepsilon \varepsilon^{\prime}}\left(F_{1} F_{4}-F_{2} F_{3}\right)\right] \\
& -2 C_{V} C_{A}\left[\frac{p_{\perp}}{\varepsilon}\left(F_{1} F_{3}+F_{2} F_{4}\right)-\frac{p_{\perp}^{\prime}}{\varepsilon^{\prime}}\left(F_{1} F_{4}+F_{2} F_{3}\right)\right], \\
& \frac{A_{23}+A_{32}}{\sin \varphi}=\left(C_{V}^{2}+C_{A}^{2}\right)\left[\frac{p_{\perp} p_{z}^{\prime}}{\varepsilon \varepsilon^{\prime}}\left(F_{1} F_{3}+F_{2} F_{4}\right)+\frac{p_{\perp}^{\prime} p_{z}}{\varepsilon \varepsilon^{\prime}}\left(F_{1} F_{4}+F_{2} F_{3}\right)\right] \\
& -2 C_{V} C_{A}\left[\frac{p_{\perp}}{\varepsilon}\left(F_{1} F_{3}-F_{2} F_{4}\right)-\frac{p_{\perp}^{\prime}}{\varepsilon^{\prime}}\left(F_{1} F_{4}-F_{2} F_{3}\right)\right], \\
& \frac{A_{23}-A_{32}}{\mathrm{i} \cos \varphi}=-\left(C_{V}^{2}+C_{A}^{2}\right)\left[\frac{p_{\perp} p_{z}^{\prime}}{\varepsilon \varepsilon^{\prime}}\left(F_{1} F_{3}-F_{2} F_{4}\right)+\frac{p_{\perp}^{\prime} p_{z}}{\varepsilon \varepsilon^{\prime}}\left(F_{1} F_{4}-F_{2} F_{3}\right)\right] \\
& +2 C_{V} C_{A}\left[\frac{p_{\perp}}{\varepsilon}\left(F_{1} F_{3}+F_{2} F_{4}\right)-\frac{p_{\perp}^{\prime}}{\varepsilon^{\prime}}\left(F_{1} F_{4}+F_{2} F_{3}\right)\right],
\end{aligned}
$$

where $p_{\perp}=\sqrt{2 n b}$ and $p_{\perp}^{\prime}=\sqrt{2 n^{\prime} b}$. The symmetric part of $A_{i j}$ corresponds to Eqs. (15) of 11. 
Let $\vartheta$ and $\alpha$ be polar and azimuthal angles of the incident neutrino momentum $\left(k_{x}=\omega \sin \vartheta \cos \alpha, k_{y}=\omega \sin \vartheta \sin \alpha\right.$, $k_{z}=\omega \cos \vartheta$ ), while $\vartheta^{\prime}$ and $\alpha^{\prime}$ be the same angles for the scattered neutrino. Then

$$
\begin{aligned}
& D=\left(1+\cos \vartheta \cos \vartheta^{\prime}\right)\left\{\left(C_{V}^{2}+C_{A}^{2}\right)\left(\varepsilon \varepsilon^{\prime}+p_{z} p_{z}^{\prime}\right)\left(F_{3}^{2}+F_{4}^{2}\right)-2 C_{V} C_{A}\left(\varepsilon^{\prime} p_{z}+\varepsilon p_{z}^{\prime}\right)\left(F_{3}^{2}-F_{4}^{2}\right)\right\} \\
& +\left(1-\cos \vartheta \cos \vartheta^{\prime}\right)\left\{\left(C_{V}^{2}+C_{A}^{2}\right)\left(\varepsilon \varepsilon^{\prime}-p_{z} p_{z}^{\prime}\right)\left(F_{1}^{2}+F_{2}^{2}\right)-\left(C_{V}^{2}-C_{A}^{2}\right)\left(F_{1}^{2}+F_{2}^{2}\right)\right. \\
& \left.+2 C_{V} C_{A}\left(\varepsilon^{\prime} p_{z}-\varepsilon p_{z}^{\prime}\right)\left(F_{1}^{2}-F_{2}^{2}\right)\right\} \\
& +\sin \vartheta \sin \vartheta^{\prime} \cos \left(\alpha-\alpha^{\prime}\right)\left\{2\left(C_{V}^{2}+C_{A}^{2}\right) p_{\perp} p_{\perp}^{\prime} F_{3} F_{4}+\left(C_{V}^{2}-C_{A}^{2}\right)\left(F_{3}^{2}+F_{4}^{2}\right)\right\} \\
& +2 \sin \vartheta \sin \vartheta^{\prime} \cos \left(\alpha+\alpha^{\prime}-2 \varphi\right)\left(C_{V}^{2}+C_{A}^{2}\right) p_{\perp} p_{\perp}^{\prime} F_{1} F_{2} \\
& -\left(\cos \vartheta+\cos \vartheta^{\prime}\right)\left\{\left(C_{V}^{2}+C_{A}^{2}\right)\left(\varepsilon^{\prime} p_{z}+\varepsilon p_{z}^{\prime}\right)\left(F_{3}^{2}+F_{4}^{2}\right)-2 C_{V} C_{A}\left(\varepsilon \varepsilon^{\prime}+p_{z} p_{z}^{\prime}\right)\left(F_{3}^{2}-F_{4}^{2}\right)\right\} \\
& -\left(\cos \vartheta-\cos \vartheta^{\prime}\right)\left\{\left(C_{V}^{2}+C_{A}^{2}\right)\left(\varepsilon \varepsilon^{\prime}-p_{z} p_{z}^{\prime}\right)\left(F_{1}^{2}-F_{2}^{2}\right)-\left(C_{V}^{2}-C_{A}^{2}\right)\left(F_{1}^{2}-F_{2}^{2}\right)+2 C_{V} C_{A}\left(\varepsilon^{\prime} p_{z}-\varepsilon p_{z}^{\prime}\right)\left(F_{1}^{2}+F_{2}^{2}\right)\right\} \\
& -\left[\sin \vartheta \cos (\alpha-\varphi)+\sin \vartheta^{\prime} \cos \left(\alpha^{\prime}-\varphi\right)\right]\left\{\left(C_{V}^{2}+C_{A}^{2}\right)\left[p_{\perp} \varepsilon^{\prime}\left(F_{1} F_{3}+F_{2} F_{4}\right)+p_{\perp}^{\prime} \varepsilon\left(F_{1} F_{4}+F_{2} F_{3}\right)\right]\right. \\
& \left.-2 C_{V} C_{A}\left[p_{\perp} p_{z}^{\prime}\left(F_{1} F_{3}-F_{2} F_{4}\right)-p_{\perp}^{\prime} p_{z}\left(F_{1} F_{4}-F_{2} F_{3}\right)\right]\right\} \\
& +\left[\sin \vartheta \cos (\alpha-\varphi)-\sin \vartheta^{\prime} \cos \left(\alpha^{\prime}-\varphi\right)\right]\left\{\left(C_{V}^{2}+C_{A}^{2}\right)\left[p_{\perp} p_{z}^{\prime}\left(F_{1} F_{3}-F_{2} F_{4}\right)+p_{\perp}^{\prime} p_{z}\left(F_{1} F_{4}-F_{2} F_{3}\right)\right]\right. \\
& \left.-2 C_{V} C_{A}\left[p_{\perp} \varepsilon^{\prime}\left(F_{1} F_{3}+F_{2} F_{4}\right)-p_{\perp}^{\prime} \varepsilon\left(F_{1} F_{4}+F_{2} F_{3}\right)\right]\right\} \\
& +\left[\cos \vartheta \sin \vartheta^{\prime} \cos \left(\alpha^{\prime}-\varphi\right)+\cos \vartheta^{\prime} \sin \vartheta \cos (\alpha-\varphi)\right]\left\{\left(C_{V}^{2}+C_{A}^{2}\right)\left[p_{\perp} p_{z}^{\prime}\left(F_{1} F_{3}+F_{2} F_{4}\right)+p_{\perp}^{\prime} p_{z}\left(F_{1} F_{4}+F_{2} F_{3}\right)\right]\right. \\
& \left.-2 C_{V} C_{A}\left[p_{\perp} \varepsilon^{\prime}\left(F_{1} F_{3}-F_{2} F_{4}\right)-p_{\perp}^{\prime} \varepsilon\left(F_{1} F_{4}-F_{2} F_{3}\right)\right]\right\} \\
& +\left[\cos \vartheta \sin \vartheta^{\prime} \cos \left(\alpha^{\prime}-\varphi\right)-\cos \vartheta^{\prime} \sin \vartheta \cos (\alpha-\varphi)\right]\left\{\left(C_{V}^{2}+C_{A}^{2}\right)\left[p_{\perp} \varepsilon^{\prime}\left(F_{1} F_{3}-F_{2} F_{4}\right)+p_{\perp}^{\prime} \varepsilon\left(F_{1} F_{4}-F_{2} F_{3}\right)\right]\right. \\
& \left.-2 C_{V} C_{A}\left[p_{\perp} p_{z}^{\prime}\left(F_{1} F_{3}+F_{2} F_{4}\right)-p_{\perp}^{\prime} p_{z}\left(F_{1} F_{4}+F_{2} F_{3}\right)\right]\right\} .
\end{aligned}
$$

One can see that $D$ and, thus, the scattering cross section depends on $\alpha-\alpha^{\prime}$ rather than on the azimutal angles $\alpha$ and $\alpha^{\prime}$ separately:

$$
\begin{aligned}
\sin (\alpha-\varphi) & =\frac{k_{\perp}^{\prime}}{q_{\perp}} \sin \left(\alpha-\alpha^{\prime}\right), \quad \sin \left(\alpha^{\prime}-\varphi\right)=\frac{k_{\perp}}{q_{\perp}} \sin \left(\alpha-\alpha^{\prime}\right), \\
\cos (\alpha-\varphi) & =\frac{1}{q_{\perp}}\left[k_{\perp}^{\prime} \cos \left(\alpha-\alpha^{\prime}\right)-k_{\perp}\right], \quad \cos \left(\alpha^{\prime}-\varphi\right)=\frac{1}{q_{\perp}}\left[k_{\perp}^{\prime}-k_{\perp} \cos \left(\alpha-\alpha^{\prime}\right)\right], \\
\cos \left(\alpha+\alpha^{\prime}-2 \varphi\right) & =\frac{1}{q_{\perp}^{2}}\left[\left(k_{\perp}^{2}+k_{\perp}^{\prime 2}\right) \cos \left(\alpha-\alpha^{\prime}\right)-2 k_{\perp} k_{\perp}^{\prime}\right],
\end{aligned}
$$

where

$$
\begin{aligned}
& k_{\perp}=\omega \sin \vartheta \\
& k_{\perp}^{\prime}=\omega^{\prime} \sin \vartheta^{\prime} \\
& q_{\perp}=\left[k_{\perp}^{2}+k_{\perp}^{\prime 2}-2 k_{\perp} k_{\perp}^{\prime} \cos \left(\alpha-\alpha^{\prime}\right)\right]^{1 / 2} .
\end{aligned}
$$

Equations (A8) cannot be used directly when $q_{\perp}=0$, i.e., when $\omega=\omega^{\prime}$ and $\alpha=\alpha^{\prime}$ simultaneously. If, however, $\alpha=\alpha^{\prime}$ one has

$$
\begin{aligned}
\sin (\alpha-\varphi) & =\sin \left(\alpha^{\prime}-\varphi\right)=0, \\
\cos (\alpha-\varphi) & =\cos \left(\alpha^{\prime}-\varphi\right)=\operatorname{sgn}\left(\omega^{\prime}-\omega\right), \\
\cos \left(\alpha+\alpha^{\prime}-2 \varphi\right) & =1,
\end{aligned}
$$

where $\operatorname{sgn}(x)=1$ for $x \geq 0$ and $\operatorname{sgn}(x)=-1$ for $x<0$.

When substituting $D$ into Eq. (12), one should set $\varepsilon^{\prime}=\varepsilon+\omega-\omega^{\prime}$ and $p_{z}^{\prime}=p_{z}+k_{z}-k_{z}^{\prime}$.

\section{APPENDIX B:}

Let us consider the neutrino scattering on non-magnetized electrons, whose quantum states are determined by the momentum $\mathbf{p}$ and helicity $s$. The differential cross section $\mathrm{d} \sigma_{\mathrm{f}}$ is calculated in the straightforward manner, using the Fermi Golden rule. After the averaging over the states of initial and final electrons, we obtain 


$$
\frac{\mathrm{d} \sigma}{\mathrm{d} \omega^{\prime} \mathrm{d} \Omega^{\prime}}=\frac{2 G_{\mathrm{F}}^{2} \omega^{\prime}}{(2 \pi)^{5} \omega}\left(C_{1} I_{1}+C_{2} I_{2}+C_{3} I_{3}\right)
$$

where $C_{1}=\left(C_{V}+C_{A}\right)^{2}, C_{2}=\left(C_{V}-C_{A}\right)^{2}, C_{3}=C_{A}^{2}-C_{V}^{2}$,

$$
\begin{aligned}
& I_{1}=I_{1}\left(k, k^{\prime}\right)=\int \frac{\mathrm{d} \mathbf{p} \mathrm{d} \mathbf{p}^{\prime}}{\varepsilon \varepsilon^{\prime}} \delta^{(4)}\left(p^{\prime}+k^{\prime}-p-k\right) f\left(1-f^{\prime}\right)(p k)\left(p^{\prime} k^{\prime}\right), \quad I_{2}=I_{2}\left(k, k^{\prime}\right)=I_{1}\left(-k^{\prime},-k\right), \\
& I_{3}=I_{3}\left(k, k^{\prime}\right)=\left(k k^{\prime}\right) \int \frac{\mathrm{d} \mathbf{p} \mathrm{d} \mathbf{p}^{\prime}}{\varepsilon \varepsilon^{\prime}} \delta^{(4)}\left(p^{\prime}+k^{\prime}-p-k\right) f\left(1-f^{\prime}\right) .
\end{aligned}
$$

Here, $p=(\varepsilon,-\mathbf{p})$ and $k=(\omega,-\mathbf{k})$ are the four-momenta of electron and neutrino before scattering, and $p^{\prime}=\left(\varepsilon^{\prime},-\mathbf{p}^{\prime}\right)$ and $k=\left(\omega^{\prime},-\mathbf{k}^{\prime}\right)$ are the 4-momenta of these particles after scattering, $f=f(\varepsilon)$ and $f^{\prime}=f\left(\varepsilon^{\prime}\right)$ are the Fermi-Dirac functions given by Eq. (9), with $\varepsilon=\sqrt{1+p^{2}}$ and $\varepsilon^{\prime}=\sqrt{1+p^{\prime 2}}$.

The four-dimensional delta-function in Eq. (B2) describes the conservation of momentum,

$$
\mathbf{p}-\mathbf{p}^{\prime}=\mathbf{q}
$$

where $\mathbf{q}=\mathbf{k}^{\prime}-\mathbf{k}$, and of energy,

$$
\varepsilon^{\prime}-\varepsilon=\Delta
$$

where $\Delta=\omega-\omega^{\prime}$. Let us first perform the integration over $\mathbf{p}^{\prime}$. We use the spherical coordinates with the polar axis along $\mathbf{q}$ to integrate over $\mathbf{p}$. This yields

$$
\begin{aligned}
& I_{1}=I_{1}\left(\omega, \omega^{\prime}, \cos \theta\right)=2 \pi \int_{0}^{\infty} \frac{p^{2} \mathrm{~d} p}{\varepsilon \varepsilon^{\prime}} f\left(1-f^{\prime}\right) \int_{-1}^{1} \mathrm{~d} \eta \delta\left(\varepsilon^{\prime}-\varepsilon-\Delta\right) I\left(\varepsilon, \varepsilon^{\prime}\right), \\
& I_{2}=I_{2}\left(\omega, \omega^{\prime}, \cos \theta\right)=I_{1}\left(-\omega^{\prime},-\omega, \cos \theta\right), \\
& I_{3}=I_{3}\left(\omega, \omega^{\prime}, \cos \theta\right)=2 \pi \omega \omega^{\prime}(1-\cos \theta) \int_{0}^{\infty} \frac{p^{2} \mathrm{~d} p}{\varepsilon \varepsilon^{\prime}} f\left(1-f^{\prime}\right) \int_{-1}^{1} \mathrm{~d} \eta \delta\left(\varepsilon^{\prime}-\varepsilon-\Delta\right) .
\end{aligned}
$$

Here, $\theta$ is the angle between $\mathbf{k}$ and $\mathbf{k}^{\prime}, \eta$ is cosine of the polar angle of the electron momentum $\mathbf{p}$, and

$$
I\left(\varepsilon, \varepsilon^{\prime}\right)=\frac{1}{2 \pi} \int_{0}^{2 \pi} \mathrm{d} \beta(p k)\left(p^{\prime} k^{\prime}\right)
$$

where $\beta$ is the azimutal angle of $\mathbf{p}$.

Furthermore, we transform the energy conserving delta-function in Eq. (B5) as:

$$
\delta\left(\varepsilon^{\prime}-\varepsilon-\Delta\right)=\frac{\varepsilon^{\prime}}{q \sqrt{\varepsilon^{2}-1}} \delta\left(\eta-\eta_{0}\right),
$$

where

$$
\eta_{0}=\frac{\varepsilon \Delta-\omega \omega^{\prime}(1-\cos \theta)}{q \sqrt{\varepsilon^{2}-1}}
$$

$\varepsilon^{\prime}=\varepsilon+\Delta$, and

$$
q=\sqrt{\omega^{2}+\omega^{\prime 2}-2 \omega \omega^{\prime} \cos \theta}
$$

The condition $\eta_{0}^{2}=1$ determines the minimal electron energy, which contributes to the integrals (B5),

$$
\varepsilon_{\min }=\frac{1}{2}\left(q \sqrt{1+\frac{2}{\omega \omega^{\prime}(1-\cos \theta)}}-\Delta\right) .
$$

Using (B7), we obtain

$$
I_{3}=2 \pi \frac{\omega \omega^{\prime}}{q}(1-\cos \theta) \int_{\varepsilon_{\min }}^{\infty} \mathrm{d} \varepsilon f\left(1-f^{\prime}\right) .
$$


Evaluation of $I_{1}$ is more complicated because of the presence of the integral (B6). The latter integral is equal to

$$
I=\varepsilon \omega \varepsilon^{\prime} \omega^{\prime}-\varepsilon \omega p_{\|}^{\prime} k_{\|}^{\prime}-\varepsilon^{\prime} \omega^{\prime} p_{\|} k_{\|}+p_{\|} k_{\|} p_{\|}^{\prime} k_{\|}^{\prime}+\frac{1}{2} p_{\mathrm{tr}}^{2} k_{\mathrm{tr}}^{2},
$$

where the subscripts "I" and "tr" denote the vector components parallel and perpendicular to q, respectively. The electron momentum components determined by conservation laws are given by

$$
\begin{aligned}
p_{\|} & =\frac{1}{2 q}\left(\varepsilon^{\prime 2}-\varepsilon^{2}-q^{2}\right), \\
p_{\|}^{\prime} & =\frac{1}{2 q}\left(\varepsilon^{\prime 2}-\varepsilon^{2}+q^{2}\right), \\
p_{\mathrm{tr}}^{2} & =\frac{1}{2 q^{2}}\left[-q^{4}+2\left(\varepsilon^{2}+\varepsilon^{\prime 2}+2\right)^{2} q^{2}-\left(\varepsilon^{2}-\varepsilon^{\prime 2}\right)^{2}\right],
\end{aligned}
$$

and the neutrino momentum components expressed through $\omega, \omega^{\prime}$ and $\cos \theta$ are,

$$
\begin{aligned}
k_{\|} & =\frac{\omega}{q}\left(\omega-\omega^{\prime} \cos \theta\right), \quad k_{\|}^{\prime}=\frac{\omega^{\prime}}{q}\left(\omega \cos \theta-\omega^{\prime}\right), \\
k_{\mathrm{tr}}^{2} & =\frac{\omega^{2} \omega^{\prime 2}}{q^{2}} \sin ^{2} \theta .
\end{aligned}
$$

Using Eqs. (B13) and (B14), we obtain

$$
I=2 \pi \frac{\omega^{2} \omega^{\prime 2}}{q^{4}}(1-\cos \theta)^{2}\left(a_{0}+a_{1} \varepsilon+a_{2} \varepsilon^{2}\right)
$$

where

$$
\begin{aligned}
& a_{0}=\omega^{2}\left[\left(\omega-\omega^{\prime} \cos \theta\right)^{2}-\frac{\omega^{\prime 2} \sin ^{2} \theta}{2}-\frac{q^{2}(1+\cos \theta)}{2 \omega^{2}(1-\cos \theta)}\right] \\
& a_{1}=\omega\left[2 \omega^{2}+\omega \omega^{\prime}(3-\cos \theta)-\omega^{\prime 2}(1+3 \cos \theta)\right] \\
& a_{2}=\omega^{2}+\omega^{\prime 2}+\omega \omega^{\prime}(3+\cos \theta) .
\end{aligned}
$$

The coefficients $a_{0}, a_{1}$ and $a_{2}$ coincide, respectively, with the coefficients $C, B$ and $A$ given by Eqs. (16)-(18) of [5] Analogously to (B11) we obtain

$$
\begin{aligned}
I_{1} & =2 \pi \frac{\omega^{2} \omega^{\prime 2}}{q^{5}}(1-\cos \theta)^{2} \\
& \times \int_{\varepsilon_{\min }}^{\infty} \mathrm{d} \varepsilon\left(a_{0}+a_{1} \varepsilon+a_{2} \varepsilon^{2}\right) f\left(1-f^{\prime}\right) .
\end{aligned}
$$

In order to integrate over electron energy in Eqs. (B11) and (B17), we use the energy conservation, (B4), and the identity

$$
f\left(1-f^{\prime}\right)=f_{\gamma}\left(-\frac{\Delta}{T}\right)\left(f-f^{\prime}\right)
$$

where

$$
f_{\gamma}(x)=\frac{1}{\exp (x)-1}
$$

is independent of electron energy $\varepsilon$. Our final result can be expressed in terms of the following integrals:

$$
\begin{aligned}
\int_{\varepsilon_{\min }}^{\infty} \mathrm{d} \varepsilon\left(f-f^{\prime}\right) & =T G_{0}, \\
\int_{\varepsilon_{\min }}^{\infty} \mathrm{d} \varepsilon \varepsilon\left(f-f^{\prime}\right) & =T^{2} G_{1}+T \varepsilon_{\min } G_{0}, \\
\int_{\varepsilon_{\min }}^{\infty} \mathrm{d} \varepsilon \varepsilon^{2}\left(f-f^{\prime}\right) & =T^{3} G_{2}+2 T^{2} \varepsilon_{\min } G_{1}+T \varepsilon_{\min }^{2} G_{0},
\end{aligned}
$$


where

$$
\begin{aligned}
G_{k} & =G_{k}\left(y, y^{\prime}\right)=F_{k}\left(y^{\prime}\right)-F_{k}(y), \\
y & =y_{0}+\frac{\Delta}{2 T}, \quad y^{\prime}=y_{0}-\frac{\Delta}{2 T}, \\
y_{0} & =\frac{1}{2 T}\left(2 \mu-q \sqrt{1+\frac{2}{\omega \omega^{\prime}(1-\cos \theta)}}\right),
\end{aligned}
$$

and

$$
F_{k}(y)=\int_{0}^{\infty} \frac{\xi^{k} \mathrm{~d} \xi}{1+\exp (\xi-y)}
$$

is a standard Fermi integral.

Finally, using Eqs. (B18) and (B20), we obtain

$$
\begin{aligned}
I_{1}= & 2 \pi \frac{\omega^{2} \omega^{\prime 2}}{q^{5}}(1-\cos \theta)^{2} f_{\gamma}\left(-\frac{\Delta}{T}\right) T \\
\times & {\left[a_{0} G_{0}+a_{1}\left(T G_{1}+\varepsilon_{\min } G_{0}\right)\right.} \\
& \left.+a_{2}\left(T^{2} G_{2}+2 T \varepsilon_{\min } G_{1}+\varepsilon_{\min }^{2} G_{0}\right)\right], \\
I_{2}= & I_{2}\left(\omega, \omega^{\prime}, \cos \theta\right)=I_{1}\left(-\omega^{\prime},-\omega, \cos \theta\right), \\
I_{3}= & 2 \pi \frac{\omega \omega^{\prime}}{q}(1-\cos \theta) f_{\gamma}\left(-\frac{\Delta}{T}\right) T G_{0} .
\end{aligned}
$$

This result coincides with Eq. (13) of [5].

\section{APPENDIX C:}

In this appendix, we transform the general expression for the NES cross section at $B=0$, introducing an arbitrary $z$-axis and integrating over orientations of the electron momenta in the corresponding perpendicular plane. Let $\beta$ and $\beta^{\prime}$ be the azimutal angles of the vectors $\mathbf{p}_{\perp}$ and $\mathbf{p}_{\perp}^{\prime}$, respectively, and $\chi$ be the angle between $\mathbf{p}_{\perp}$ and $\mathbf{p}_{\perp}^{\prime}$. It is evident that

$$
\mathrm{d} \mathbf{p} \mathrm{d} \mathbf{p}^{\prime}=\frac{1}{4} \mathrm{~d} p_{z} \mathrm{~d} p_{z}^{\prime} \mathrm{d} p_{\perp}^{2} \mathrm{~d} p_{\perp}^{\prime 2} \mathrm{~d} \chi \mathrm{d} \beta .
$$

¿From Eqs. (B1) and (B2) we obtain

$$
\frac{\mathrm{d} \sigma}{\mathrm{d} \omega^{\prime} \mathrm{d} \Omega^{\prime}}=\frac{G_{\mathrm{F}}^{2} \omega^{\prime}}{2(2 \pi)^{5} \omega} \iint_{0}^{\infty} \mathrm{d} p_{\perp}^{2} \mathrm{~d} p_{\perp}^{\prime 2} \int_{-\infty}^{+\infty} \frac{\mathrm{d} p_{z}}{\varepsilon \varepsilon^{\prime}} \delta\left(\varepsilon^{\prime}+\omega^{\prime}-\varepsilon-\omega\right) f\left(1-f^{\prime}\right) R,
$$

where

$$
\begin{aligned}
R & =R_{1}+R_{2}+R_{3}, \\
R_{1} & =C_{1} \int_{0}^{2 \pi} \mathrm{d} \chi \int_{0}^{2 \pi} \mathrm{d} \beta \delta\left(\mathbf{p}_{\perp}^{\prime}+\mathbf{k}_{\perp}^{\prime}-\mathbf{p}_{\perp}-\mathbf{k}_{\perp}\right)(p k)\left(p^{\prime} k^{\prime}\right), \\
R_{2} & =C_{2} \int_{0}^{2 \pi} \mathrm{d} \chi \int_{0}^{2 \pi} \mathrm{d} \beta \delta\left(\mathbf{p}_{\perp}^{\prime}+\mathbf{k}_{\perp}^{\prime}-\mathbf{p}_{\perp}-\mathbf{k}_{\perp}\right)\left(p k^{\prime}\right)\left(p^{\prime} k\right), \\
R_{3} & =C_{3}\left(k k^{\prime}\right) \int_{0}^{2 \pi} \mathrm{d} \chi \int_{0}^{2 \pi} \mathrm{d} \beta \delta\left(\mathbf{p}_{\perp}^{\prime}+\mathbf{k}_{\perp}^{\prime}-\mathbf{p}_{\perp}-\mathbf{k}_{\perp}\right) .
\end{aligned}
$$

Our aim is to integrate over $\chi$ and $\beta$ in Eq. (C3). We use the conservation of the transverse electron momentum, which implies

$$
\mathbf{p}_{\perp}-\mathbf{p}_{\perp}^{\prime}=\mathbf{q}_{\perp}
$$


where $\mathbf{q}_{\perp}=\mathbf{k}_{\perp}^{\prime}-\mathbf{k}_{\perp}$. Combined with the relation $\beta^{\prime}=\beta+\chi$, Eq. (C4) leads to the following equations for $\cos \beta$ and $\sin \beta$ :

$$
\begin{aligned}
\left(p_{\perp}-p_{\perp}^{\prime} \cos \chi\right) \cos \beta+p_{\perp}^{\prime} \sin \chi \sin \beta & =q_{\perp} \cos \varphi, \\
-p_{\perp}^{\prime} \sin \chi \cos \beta+\left(p_{\perp}-p_{\perp}^{\prime} \cos \chi\right) \sin \beta & =q_{\perp} \sin \varphi,
\end{aligned}
$$

where $\varphi$ is the azimutal angle of $\mathbf{q}_{\perp}$. ¿From (C5) we obtain

$$
\begin{aligned}
& \cos \beta=\frac{1}{q_{\perp}}\left[\left(p_{\perp}-p_{\perp}^{\prime} \cos \chi\right) \cos \varphi-p_{\perp}^{\prime} \sin \chi \sin \varphi\right], \\
& \sin \beta=\frac{1}{q_{\perp}}\left[p_{\perp}^{\prime} \sin \chi \cos \varphi+\left(p_{\perp}-p_{\perp}^{\prime} \cos \chi\right) \sin \varphi\right],
\end{aligned}
$$

and

$$
\begin{aligned}
& \cos \beta^{\prime}=\frac{1}{q_{\perp}}\left[\left(p_{\perp} \cos \chi-p_{\perp}^{\prime}\right) \cos \varphi-p_{\perp} \sin \chi \sin \varphi\right] \\
& \sin \beta^{\prime}=\frac{1}{q_{\perp}}\left[p_{\perp} \sin \chi \cos \varphi+\left(p_{\perp} \cos \chi-p_{\perp}^{\prime}\right) \sin \varphi\right] .
\end{aligned}
$$

¿From squared Eq. ( $\overline{\mathrm{C} 4}$ ), we determine two possible "resonant" values of $\chi$ which contribute to the integrals over the directions of $\mathbf{p}_{\perp}$ and $\mathbf{p}_{\perp}$ :

$$
\begin{aligned}
\cos \chi_{\lambda} & =\frac{p_{\perp}^{2}+p_{\perp}^{2}-q_{\perp}^{2}}{2 p_{\perp} p_{\perp}^{\prime}}, \\
\sin \chi_{\lambda} & =(-1)^{\lambda} \frac{\sqrt{\left(p_{1}^{2}-q_{\perp}^{2}\right)\left(q_{\perp}^{2}-p_{2}^{2}\right)}}{2 p_{\perp} p_{\perp}^{\prime}},
\end{aligned}
$$

where $\lambda=1,2$ and $p_{1,2}=p_{\perp}^{\prime} \pm p_{\perp}$. We can rewrite the delta-function in Eqs. (C3) as

$$
\begin{aligned}
\delta\left(\mathbf{p}_{\perp}^{\prime}+\mathbf{k}_{\perp}^{\prime}-\mathbf{p}_{\perp}-\mathbf{k}_{\perp}\right) & =\frac{2}{\sqrt{\left(p_{1}^{2}-q_{\perp}^{2}\right)\left(q_{\perp}^{2}-p_{2}^{2}\right)}} \\
& \times \sum_{\lambda=1,2} \delta\left(\chi-\chi_{\lambda}\right) \delta\left(\beta-\beta_{\lambda}\right),
\end{aligned}
$$

where the angles $\beta_{\lambda}$ are determined by Eqs. (C6) and (C7) with $\chi=\chi_{\lambda}$. This yields

$$
R_{3}=\frac{4 C_{3} \omega \omega^{\prime} Q_{3}}{\sqrt{\left(p_{1}^{2}-q_{\perp}^{2}\right)\left(q_{\perp}^{2}-p_{2}^{2}\right)}}
$$

where

$$
Q_{3}=1-\cos \vartheta \cos \vartheta^{\prime}-\sin \vartheta \sin \vartheta^{\prime} \cos \left(\alpha-\alpha^{\prime}\right) .
$$

In this case $\vartheta$ and $\vartheta^{\prime}$ are the polar angles, and $\alpha$ and $\alpha^{\prime}$ are the azimuthal angles of $\mathbf{k}$ and $\mathbf{k}^{\prime}$, respectively.

The expression for $R_{1}$ contains the quantity

$$
(p k)\left(p^{\prime} k^{\prime}\right)=\left(\varepsilon \omega-p_{z} k_{z}-\mathbf{p}_{\perp} \mathbf{k}_{\perp}\right)\left(\varepsilon^{\prime} \omega^{\prime}-p_{z}^{\prime} k_{z}^{\prime}-\mathbf{p}_{\perp}^{\prime} \mathbf{k}_{\perp}^{\prime}\right),
$$

in which $\mathbf{k}_{\perp} \mathbf{p}_{\perp}$ and $\mathbf{k}_{\perp}^{\prime} \mathbf{p}_{\perp}^{\prime}$ depend on the integration variable $\chi$. Using Eqs. (C6) and (C7), we can explicitly express these terms through $\chi$,

$$
\begin{aligned}
& \mathbf{p}_{\perp} \mathbf{k}_{\perp}=\frac{p_{\perp} k_{\perp}}{q_{\perp}}\left[\left(p_{\perp}-p_{\perp}^{\prime} \cos \chi\right) \cos (\alpha-\varphi)+p_{\perp}^{\prime} \sin \chi \sin (\alpha-\varphi)\right], \\
& \mathbf{p}_{\perp}^{\prime} \mathbf{k}_{\perp}^{\prime}=\frac{p_{\perp}^{\prime} k_{\perp}^{\prime}}{q_{\perp}}\left[\left(p_{\perp} \cos \chi-p_{\perp}^{\prime}\right) \cos \left(\alpha^{\prime}-\varphi\right)+p_{\perp} \sin \chi \sin \left(\alpha^{\prime}-\varphi\right)\right] .
\end{aligned}
$$

This gives 


$$
R_{1}=\frac{4 C_{1} \omega \omega^{\prime} Q_{1}}{\sqrt{\left(p_{1}^{2}-q_{\perp}^{2}\right)\left(q_{\perp}^{2}-p_{2}^{2}\right)}}
$$

where

$$
\begin{aligned}
\omega \omega^{\prime} Q_{1} & =\left(\varepsilon \omega-p_{z} k_{z}\right)\left(\varepsilon^{\prime} \omega^{\prime}-p_{z}^{\prime} k_{z}^{\prime}\right) \\
& -\frac{p_{\perp}^{\prime} k_{\perp}^{\prime}}{q_{\perp}}\left(\varepsilon \omega-p_{z} k_{z}\right)\left(p_{\perp} \cos \chi-p_{\perp}^{\prime}\right) \cos \left(\alpha^{\prime}-\varphi\right)-\frac{p_{\perp} k_{\perp}}{q_{\perp}}\left(\varepsilon^{\prime} \omega^{\prime}-p_{z}^{\prime} k_{z}^{\prime}\right)\left(p_{\perp}-p_{\perp}^{\prime} \cos \chi\right) \cos (\alpha-\varphi) \\
& +\frac{p_{\perp} p_{\perp}^{\prime} k_{\perp} k_{\perp}^{\prime}}{q_{\perp}^{2}}\left[\left(p_{\perp}-p_{\perp}^{\prime} \cos \chi\right)\left(p_{\perp} \cos \chi-p_{\perp}^{\prime}\right) \cos (\alpha-\varphi) \cos \left(\alpha^{\prime}-\varphi\right)+p_{\perp} p_{\perp}^{\prime} \sin ^{2} \chi \sin (\alpha-\varphi) \sin \left(\alpha^{\prime}-\varphi\right)\right]
\end{aligned}
$$

$\cos \chi$ and $\sin \chi$ are given by Eq. (C8). Notice, that the terms proportional to $\sin \chi$ do not contribute to $Q_{1}$ since they cancel out after summation over $\lambda=1,2$. Introducing $\cos \left(\alpha-\alpha^{\prime}\right)$ and $\cos \left(\alpha+\alpha^{\prime}-2 \varphi\right)$ and using Eq. (C8), we obtain

$$
\begin{aligned}
Q_{1} & =\varepsilon \varepsilon^{\prime}+p_{z} p_{z}^{\prime} \cos \vartheta \cos \vartheta^{\prime}-\varepsilon p_{z}^{\prime} \cos \vartheta^{\prime}-\varepsilon^{\prime} p_{z} \cos \vartheta \\
& -\left(\varepsilon-p_{z} \cos \vartheta\right) \sin \vartheta^{\prime} \cos \left(\alpha^{\prime}-\varphi\right) \frac{p_{\perp}^{2}-p_{\perp}^{\prime 2}-q_{\perp}^{2}}{2 q_{\perp}}-\left(\varepsilon^{\prime}-p_{z}^{\prime} \cos \vartheta^{\prime}\right) \sin \vartheta \cos (\alpha-\varphi) \frac{p_{\perp}^{2}-p_{\perp}^{2}+q_{\perp}^{2}}{2 q_{\perp}} \\
& +\sin \vartheta \sin \vartheta^{\prime} \cos \left(\alpha+\alpha^{\prime}-2 \varphi\right) \frac{\left(p_{\perp}^{2}-p_{\perp}^{\prime 2}\right)^{2}-\left(p_{\perp}^{2}+p_{\perp}^{\prime 2}\right) q_{\perp}^{2}}{4 q_{\perp}^{2}}+\sin \vartheta \sin \vartheta^{\prime} \cos \left(\alpha-\alpha^{\prime}\right) \frac{p_{\perp}^{2}+p_{\perp}^{\prime 2}-q_{\perp}^{2}}{4} .
\end{aligned}
$$

In analogy with (C14), we have

$$
R_{2}=\frac{4 C_{1} \omega \omega^{\prime} Q_{2}}{\sqrt{\left(p_{1}^{2}-q_{\perp}^{2}\right)\left(q_{\perp}^{2}-p_{2}^{2}\right)}}
$$

where $Q_{2}$ is obtained from $Q_{1}$ by replacing $\vartheta^{\prime} \leftrightarrow \vartheta, \alpha^{\prime} \leftrightarrow \alpha$. The final expression for the NES cross section reads

$$
\frac{\mathrm{d} \sigma}{\mathrm{d} \omega^{\prime} \mathrm{d} \Omega^{\prime}}=\frac{2 G_{\mathrm{F}}^{2} \omega^{\prime 2}}{(2 \pi)^{5}} \iint \frac{\mathrm{d} p_{\perp}^{2} \mathrm{~d} p_{\perp}^{\prime 2}}{\sqrt{\left(p_{1}^{2}-q_{\perp}^{2}\right)\left(q_{\perp}^{2}-p_{2}^{2}\right)}} \int_{-\infty}^{+\infty} \frac{\mathrm{d} p_{z}}{\varepsilon \varepsilon^{\prime}} \delta\left(\varepsilon^{\prime}+\omega^{\prime}-\varepsilon-\omega\right) f\left(1-f^{\prime}\right) Q,
$$

where the integration domain over the transverse electron momenta is restricted by the inequalities $p_{2} \geq q_{\perp}$ and $\left|p_{1}\right| \leq q_{\perp}$. The quantity $Q=C_{1} Q_{1}+C_{2} Q_{2}+C_{3} Q_{3}$ is explicitly given by the following expression:

$$
\begin{aligned}
Q & =\left(1+\cos \vartheta \cos \vartheta^{\prime}\right)\left(C_{V}^{2}+C_{A}^{2}\right)\left(\varepsilon \varepsilon^{\prime}+p_{z} p_{z}^{\prime}\right)+\left(1-\cos \vartheta \cos \vartheta^{\prime}\right)\left[\left(C_{V}^{2}+C_{A}^{2}\right)\left(\varepsilon \varepsilon^{\prime}-p_{z} p_{z}^{\prime}\right)-\left(C_{V}^{2}-C_{A}^{2}\right)\right] \\
& +\sin \vartheta \sin \vartheta^{\prime} \cos \left(\alpha-\alpha^{\prime}\right)\left[\left(C_{V}^{2}+C_{A}^{2}\right) \frac{p_{\perp}^{2}+p_{\perp}^{\prime 2}-q_{\perp}^{2}}{2}+\left(C_{V}^{2}-C_{A}^{2}\right)\right] \\
& +\sin \vartheta \sin \vartheta^{\prime} \cos \left(\alpha+\alpha^{\prime}-2 \varphi\right)\left(C_{V}^{2}+C_{A}^{2}\right) \frac{\left(p_{\perp}^{2}-p_{\perp}^{\prime 2}\right)^{2}-\left(p_{\perp}^{2}+p_{\perp}^{\prime 2}\right) q_{\perp}^{2}}{2 q_{\perp}^{2}} \\
& -\left(\cos \vartheta+\cos \vartheta^{\prime}\right)\left(C_{V}^{2}+C_{A}^{2}\right)\left(\varepsilon^{\prime} p_{z}+\varepsilon p_{z}^{\prime}\right)-\left(\cos \vartheta-\cos \vartheta^{\prime}\right) 2 C_{V} C_{A}\left(\varepsilon^{\prime} p_{z}-\varepsilon p_{z}^{\prime}\right) \\
& -\left[\sin \vartheta \cos (\alpha-\varphi)+\sin \vartheta^{\prime} \cos \left(\alpha^{\prime}-\varphi\right)\right]\left(C_{V}^{2}+C_{A}^{2}\right)\left[\varepsilon^{\prime} \frac{p_{\perp}^{2}-p_{\perp}^{\prime 2}+q_{\perp}^{2}}{2 q_{\perp}}+\varepsilon \frac{p_{\perp}^{2}-p_{\perp}^{\prime 2}-q_{\perp}^{2}}{2 q_{\perp}}\right] \\
& -\left[\sin \vartheta \cos (\alpha-\varphi)-\sin \vartheta^{\prime} \cos \left(\alpha^{\prime}-\varphi\right)\right] 2 C_{V} C_{A}\left[\varepsilon^{\prime} \frac{p_{\perp}^{2}-p_{\perp}^{\prime 2}+q_{\perp}^{2}}{2 q_{\perp}}-\varepsilon \frac{p_{\perp}^{2}-p_{\perp}^{\prime 2}-q_{\perp}^{2}}{2 q_{\perp}}\right] \\
& +\left[\cos \vartheta \sin \vartheta^{\prime} \cos \left(\alpha^{\prime}-\varphi\right)+\cos \vartheta^{\prime} \sin \vartheta \cos (\alpha-\varphi)\right]\left(C_{V}^{2}+C_{A}^{2}\right)\left[p_{z}^{\prime} \frac{p_{\perp}^{2}-p_{\perp}^{\prime 2}+q_{\perp}^{2}}{2 q_{\perp}}+p_{z} \frac{p_{\perp}^{2}-p_{\perp}^{\prime 2}-q_{\perp}^{2}}{2 q_{\perp}}\right] \\
& -\left[\cos \vartheta \sin \vartheta^{\prime} \cos \left(\alpha^{\prime}-\varphi\right)-\cos \vartheta^{\prime} \sin \vartheta \cos (\alpha-\varphi)\right] 2 C_{V} C_{A}\left[p_{z}^{\prime} \frac{p_{\perp}^{2}-p_{\perp}^{\prime 2}+q_{\perp}^{2}}{2 q_{\perp}}-p_{z} \frac{p_{\perp}^{2}-p_{\perp}^{2}-q_{\perp}^{2}}{2 q_{\perp}}\right] .
\end{aligned}
$$

[1] J. Cooperstein, Phys. Reports 163, 95 (1988) 
[2] S.W. Bruenn, Astrophys. J. Suppl. 58, 771 (1985)

[3] S.W. Bruenn, Astrophys. Space Sci. 143, 15 (1988)

[4] E.S. Myra, Phys. Reports 163, 127 (1988)

[5] A. Mezzacappa and S.W. Bruenn, Astrophys. J. 410, 740 (1993)

[6] J. LeBlanc and J.R. Wilson, Astrophys. J. 161, 541 (1970)

[7] G.S. Bisnovatyi-Kogan, Yu.P. Popov and A.A. Samochin, Astrophys. Space Sci. 41, 287 (1976)

[8] E. Mueller and H. Hillebrandt, Astron. Astrophys. 80, 147 (1979)

[9] W.R. Yueh and R. Buchler, Astrophys. Sp. Sci. 39, 429 (1976)

[10] P. Schinder and Shapiro, Astrophys. J. Suppl. 50, 23 (1982)

[11] A.D. Kaminker, K.P. Levenfish, D.G. Yakovlev, P. Amsterdamski and P. Haensel, Phys. Rev. D 46, 3256 (1992)

[12] A.D. Kaminker and D.G. Yakovlev, Teor. Mat. Fiz. 49, 248 (1981)

[13] D.G. Yakovlev and R. Tschaepe, Astron. Nachr. 302, 167 (1981)

[14] V.G. Bezchastnov and G.G. Pavlov, Astrophys. Space Sci. 178, 1 (1991)

[15] D.L. Tubbs and D.N. Schramm, Astrophys. J. 201, 467 (1975)

[16] D.G. Yakovlev and V.A. Urpin, Soviet Astrofizika 24, 303 (1980)

[17] D. Lai and S.L. Shapiro, Astrophys. J. 383, 745 (1991)

FIG. 1. The differential neutrino-electron scattering cross section (in units of $\sigma_{0}=4 G_{\mathrm{F}}^{2} / \pi=1.764 \times 10^{-44} \mathrm{~cm}^{2}$ ) as the function of the energy of the incident neutrino (top panel) and the corresponding dependence of the parameter $\xi$ (bottom panel). The horizontal lines show the thresholds $\xi_{1}$ associated with the electron transition from (to) the ground Landau level for different harmonics numbers $\nu$, and dashed vertical lines label the square-root features of the cross section corresponding to the thresholds. The symbols A1, A2, B1 and B2 denote the domains of the cross section, described by Eqs. (18), (21), (24) and (29), respectively. The value of $\mu$ corresponds to $\rho Z / A=1.945 \times 10^{11} \mathrm{~g} \mathrm{~cm}^{-3}$. The part of the cross section inside the bar in the left bottom corner of the top plot is shown separately in Fig. 2.

FIG. 2. The behaviour of the cross section near the boundary of the domains B1 and B2. The peaks labeled by the square and triangle correspond to the transitions $n=2 \rightarrow n^{\prime}=1$ and $n=1 \rightarrow n^{\prime}=1$, respectively.

FIG. 3. The influence of the magnetic field on the NES cross section. The left panel shows the cross-section as the function of the incident neutrino energy at $\omega^{\prime}=15$. The right panel presents the dependence of the cross section on the energy of the scattered neutrino at $\omega=15$. Dashed lines show the cross sections at zero magnetic field. The calculations were done for $\mu=60$ (which corresponds to $\rho Z / A \simeq 1.9 \times 10^{11} \mathrm{~g} \mathrm{~cm}^{-3}$ ), and $T=3$.

FIG. 4. The cross section for the case when the scattered neutrino propagates in the plane "k-B" and the angle of scattering (between $\mathbf{k}^{\prime}$ and $\mathbf{k}$ ) is $\theta=90^{\circ}$. The scattering cross sections are shown as the functions of $\omega^{\prime} / \omega$, at different angles $\vartheta$ between $\mathbf{k}$ and $\mathbf{B}$. Dashed curves correspond to the zero field limit.

FIG. 5. The same as in Figure 4, for the case when the scattered neutrino propagates transverse to the plane "k-B".

FIG. 6. The effect of the replacement $\vartheta^{\prime} \leftrightarrow \vartheta$ on the scattering cross section in strong magnetic field. 